\title{
Asymptotically Optimal Encodings of Range Data Structures for Selection and Top- $k$ Queries*
}

\author{
Roberto Grossi ${ }^{\dagger} \quad$ John Iacono ${ }^{\ddagger} \quad$ Gonzalo Navarro ${ }^{\S} \quad$ Rajeev Raman \\ S. Rao Sattill
}

\begin{abstract}
Given an array $A[1, n]$ of elements with a total order, we consider the problem of building a data structure that solves two queries: $(a)$ selection queries receive a range $[i, j]$ and an integer $k$ and return the position of the $k$ th largest element in $A[i, j] ;(b)$ top- $k$ queries receive $[i, j]$ and $k$ and return the positions of the $k$ largest elements in $A[i, j]$. These problems can be solved in optimal time, $O(1+\lg k / \lg \lg n)$ and $O(k)$, respectively, using linear-space data structures.

We provide the first study of the encoding data structures for the above problems, where $A$ cannot be accessed at query time. Several applications are interested in the relative order of the entries of $A$, and their positions, rather their actual values, and thus we do not need to keep $A$ at query time. In those cases, encodings save storage space: we first show that any encoding answering such queries requires $n \lg k-O(n+k \lg k)$ bits of space; then, we design encodings using $O(n \lg k)$ bits, that is, asymptotically optimal up to constant factors, while preserving optimal query time.
\end{abstract}

\section{Introduction}

A frequent problem in data and log mining applications is to find highest or lowest values in a range of a stream: the coldest days in a time period, peaks in the stock market, most popular terms in Twitter, most frequent queries in Google, and so on. As a less obvious scenario, consider autocompletion search in databases $[22,24]$. As the user types in a query, the system presents the $k$ most highly scoring (i.e., the most popular) completions of the text entered so far, chosen from a lexicon of phrases. Viewing the lexicon as a sorted sequence of strings with scores stored in an array $A$, the system maintains the range $[i, j]$ of the phrases prefixed by text typed in so far, and chooses the strings with the $k$ highest scores in $A[i, j]$. Similarly, in Web search engines, $A$ could contain the sequence of PageRank values of the pages in an inverted list sorted by URL. Then we

\footnotetext{
*Early partial versions of this article appeared in Proc. ESA 2013 and Proc. FSTTCS 2014. Grossi partially funded by MIUR PRIN 2012C4E3KT national research project; Navarro funded in part by Millennium Nucleus Information and Coordination in Networks ICM/FIC P10-024F; Satti partly supported by Basic Science Research Program through the National Research Foundation of Korea (NRF) funded by the Ministry of Education, Science and Technology (Grant number 2012-0008241).

${ }^{\dagger}$ Department of Informatics, University of Pisa, Italy

${ }^{\ddagger}$ Department of Computer Science and Engineering, Polytechnic Institute of New York University, USA

${ }^{\S}$ Department of Computer Science, University of Chile, Chile

『Department of Computer Science, University of Leicester, UK

" School of Computer Science and Engineering, Seoul National University, Korea
} 
could efficiently retrieve the $k$ most highly ranked pages that contain a query term, restricted to a range of page identifiers (which can model a domain of any granularity). The problem is, again, to find the $k$ highest values in a range $A[i, j]$. Directly finding the $k$ th highest value may also be of interest. For example, in interfaces that show the first $k$ results and then, upon user request, the next $k$, it is useful to obtain the $(k+1)$ th to $2 k$ th results without having to obtain the first $k$ results again.

The research work presented in this paper is motivated by the observation that, in these examples, the actual contents of $A$ are not interesting by themselves (e.g., the scores are not reported). All we need is to find the positions in $A$ where the highest values occur in a range. Hence storage of the contents of $A$ could be avoided if we had a way to find those highest values without accessing $A$ at query time.

We now formalize the problem of interest. Consider an array $A[1, n]$ of integers, reals, or in general any totally sorted universe. We are interested in the following two queries on $A$ :

1. Selection queries: $\operatorname{sel}(i, j, k)$ returns the position of the $k$ th largest value in range $A[i, j]$, for any given $1 \leq i \leq j \leq n$ and $1 \leq k \leq j-i+1$.

2. Top- $k$ queries: top $(i, j, k)$ returns the positions of the $k$ largest values in $A[i, j]$, in sorted order of value, for any given $1 \leq i \leq j \leq n$ and $1 \leq k \leq j-i+1$.

Since these queries are sensitive only to the relative order between elements of $A$, and not to the actual values, we can replace the values in $A$ by their rank (i.e., their position after sorting $A$ in increasing order, breaking ties arbitrarily), and all the sel $(\cdot)$ and top $(\cdot)$ queries will return correct answers. Thus, in the sequel, we will consider that $A$ is already a permutation of $[n]$ without loss of generality.

While optimal-time solutions exist for implementing those two queries, in this article we are interested in a kind of data structures called an encoding. An encoding is a data structure that, after preprocessing $A$, can answer queries on $A$ without accessing $A$ itself. Encodings are interesting when they use less space than that necessary to represent $A$ (let us call it $|A|$ ). Otherwise, any data structure allowed to use $O(|A|)$ space could be modified to contain a copy of $A$ inside, and then trivially become an encoding. Thus, interesting encodings cannot, by definition, recover all the values of $A$, but they can still answer the predefined queries for which they have been designed.

In our case, since $A$ stores a permutation of $[n]$ and thus its storage requires $|A| \geq \lg _{2} n$ ! = $\Theta(n \lg n)$ bits, we will be interested in encodings that use $o(n \lg n)$ bits. Such encodings are useful when the values in $A$ are intrinsically uninteresting and only the indices where the sel $(\cdot)$ or top $(\cdot)$ values occur are sufficient, which is the case for the applications mentioned before.

Contributions. Since encodings do not access the data in $A$, a first question is what is the minimum size an encoding must have in order to answer the desired queries, irrespectively of the query time. In Section 3 we prove with a simple argument that any encoding solving either sel(·) or top $(\cdot)$ queries requires $n \lg k-O(n+k \lg k)$ bits of space, even if we restrict the query ranges to one-sided queries, of the form $A[1, j]$.

This shows that there are inherent limitations in space saving: we cannot hope to have an interesting encoding that works for any value of $k$, because values where $\lg k=\Theta(\lg n)$ would require encodings of $\Theta(n \lg n)$ bits, which are not interesting according to our definition. Still the challenge is to find encodings for some given maximum $k$ value, $\kappa$, which handle queries for any $1 \leq k \leq \kappa$. Thus we can aim at encodings of size $O(n \lg \kappa)=o(n \lg n)$ when $\lg \kappa=o(\lg n)$. 
The core of our research work aims at an encoding that, in $O(n \lg \kappa)$ bits of space, solves queries $\operatorname{sel}(i, j, k)$ in time $O(1+\lg k / \lg \lg n)$, for any $1 \leq k \leq \kappa$. The space is optimal up to constant factors, whereas the time is optimal for any structure using $O(n$ polylog $n)$ space [23]. Then we show how the structure for sel(.) can also be used to solve top $(i, j, k)$ queries in optimal time, $O(k)$. As a special case, we also show that sel(·) queries can break the time lower bound for $\operatorname{sel}(1, j, \kappa)$ queries, that is, if they are one-sided and work only for $k=\kappa$ fixed at construction time. All our time results hold on a RAM machine with words of $w=\Theta(\lg n)$ bits.

Related work. The sel(.) and top $(\cdot)$ query problems are a natural extension of the well-known range maximum query (RMQ) problem, which corresponds to both $\operatorname{sel}(\cdot)$ and top $(\cdot)$ with $k=1$ : namely, query $\mathrm{rmq}(i, j)$ looks for the position of the largest value in $A[i, j]$. The problem of encoding RMQs is well studied [12,14,32]. Fischer and Heun [14] gave an encoding of $A$ that uses $2 n+o(n)$ bits and answers RMQs in $O(1)$ time; their space bound is asymptotically optimal to within lower-order terms. The case $k=2$ was studied more recently by Davoodi et al. [11], obtaining $3.272 n+o(n)$ bits of space and $O(1)$ time.

We are not aware of any previous work on sel(.) or top $(\cdot)$ encoding for general $k$. After the conference versions of this article appeared [20,28], Gawrychowski and Nicholson [18] found the exact main term in the lower bound for these encodings, $n \lg k+n(k+1) \lg (1+1 / k)$, which is between $n \lg k+n / \ln 2$ and $n \lg k+\frac{k+1}{k} n / \ln 2$. This bound refines ours in the lower-order term, $O(n)$. They also build an encoding using optimal space up to lower-order terms. This encoding supports the queries, but not efficiently (i.e., it needs $\Omega(n)$ time), thus it is closer to a storage method than to a data structure with optimal query time. Their most recent version [17] contains an encoding using $1.5 n \lg \kappa-\Theta(n)$ bits, which solves queries top $(i, j, \kappa)$ and $\operatorname{sel}(i, j, \kappa)$, for $\kappa$ fixed at construction, in time $O\left(\kappa^{6} \lg ^{2} n \omega(1)\right)$. This time is now sublinear, but still far from optimal.

The non-encoding version of the sel(.) query problem has recently been studied intensively $[7,9,15,16,23]$, always using linear space (i.e., $O(n \lg n)$ bits). Gagie et al. $[15,16]$ solved the problem in $O(\lg n)$ time for any $k$, using a wavelet tree representation of $A$. Brodal and Jørgensen [8] reduced the time to $O(\lg n / \lg \lg n)$, with a structure similar to a multi-ary wavelet tree. Jørgensen and Larsen [23] obtained a query time of $O(\lg k / \lg \lg n+\lg \lg n)$, finally improved to $O(1+\lg k / \lg \lg n)$ by Chan and Wilkinson [9]. ${ }^{1}$ These last two solutions build on an idea called shallow cuttings [25], which allows one to decompose the general problem into $O(n / k)$ carefully chosen problems of size $O(k)$, and then using Brodal and Jørgensen's structure [8] on those subproblems. We will also use shallow cuttings in our solutions.

Jørgensen and Larsen [23] introduced the $\kappa$-capped range selection problem, where a parameter $\kappa$ is provided at preprocessing time, and the data structure only supports selection for ranks $1 \leq k \leq \kappa$ (as explained, interesting encodings can only solve this $\kappa$-capped version of the problem). They showed that even the one-sided $\kappa$-capped range selection problem requires query time $\Omega(\lg k / \lg \lg n)$ for structures using $O(n$ polylog $n)$ words; therefore the result of Chan and Wilkinson is the best possible for that space. This also shows that our faster results for one-sided queries are possible only because the structures only solve queries with $k=\kappa$.

It is worth noting that the data structures presented in this article are not merely a succinct implementation of the shallow cutting idea employed by Chan and Wilkinson [9] to obtain their

\footnotetext{
${ }^{1}$ Chan and Wilkinson claim a bound of $O\left(1+\lg _{w} k\right)$ for the "trans-dichotomous" model with word $\operatorname{size} w=\Omega(\lg n)$. This is, however, based on an incorrect application of a result of Grossi et al. [21]; the proof presented in their paper [9] only yields a time bound of $O(1+\lg k / \lg \lg n)$ (B. T. Wilkinson, personal communication).
} 
optimal time. As their solution requires access to the array $A$ at query time, we must address the simultaneous problems of reducing the space to asymptotically optimal, preserving optimal query time, and avoiding to access $A$ during a query.

In the non-encoding model, the top $(\cdot)$ query problem could be solved with our optimal-time sel $(\cdot)$ solution at hand (see, e.g., Muthukrishnan [27]). We first obtain the $k$ th value, $v$, and then use an RMQ data structure on $A$ : We compute $p=\mathrm{rmq}(i, j)$, report it, and then continue recursively on the intervals $A[i, p-1]$ and $A[p+1, j]$, stopping as soon as we obtain values smaller than $v$. This takes the optimal $O(k)$ time. Note, however, that this idea cannot be directly used in the encoding model because the value $v$ is not available and thus cannot be exploited as mentioned above. It also does not deliver the results in sorted order. Brodal et al. [6] gave linear-space data structures to retrieve the top- $k$ results in order in time $O(k)$, even in online form where each new result is delivered in $O(1)$ time, without knowing $k$ in advance. However, these data structures are not encodings as they require the explicit values of $A$.

Problem of independent interest. We single out a problem that could have other applications, and that arises as a subproblem in our encoding (see Section 5.1). Consider an array $Y[1, t]$ of $t$ elements under a total order. Given a construction-time parameter $\ell$, the purpose is to design an encoding to solve the following queries having any $1 \leq j \leq n$ and $1 \leq d \leq \ell$ as input (recall that we cannot access $Y$ at query time).

1. Next-larger queries: next-larger $(j, d)$ returns the position of the $d$ th left-to-right value in $Y[j+1, t]$ that is strictly larger than $Y[j]$.

2. Previous-larger queries: prev-larger $(j, d)$ returns the position of the $d$ th right-to-left value in $Y[1, j-1]$ that is strictly larger than $Y[j]$.

The above queries return a special value 0 when the wanted position does not exist. In Section 5.1 we describe an encoding that answers queries in time $O(d)$, using $O(\ell t)$ bits of space. ${ }^{2}$ This is mostly interesting for low values of $\ell$, generalizing the existing structures that solve the case $\ell=1$ [13]. Previous-smaller and next-smaller queries are obvious variants that can be solved similarly. In a conference version [20, Sec. 3.1] we showed how this encoding can be used to solve top $(i, j, k)$ queries for any $1 \leq k \leq \kappa$, using $O(\kappa n)$ bits and $O\left(k^{2}\right)$ time, but this is subsumed in space and time by our better top $(\cdot)$ solutions in this article.

Paper organization. The paper is organized as follows. In Section 2 we give an overview of the known succinct data strucures that we employ for our encodings. We present the lower bound on the space required by any encondings for our problem in Section 3. After that, we describe our general approach and relate it to the existing solutions based on shallow cuttings in Section 4, and give its succinct implementation in Section 5: in these sections, we pose a number of algorithmic challenges that are solved in Sections 6-8. Finally, we describe an encoding for the special case of one-sided queries in Section 9 and draw our conclusions in Section 10.

\footnotetext{
${ }^{2}$ Each query to our encoding can actually report all the $d$ (left-to-right or right-to-left, respectively) values in time $O(d)$. The reason is that to answer, say, next-larger $(j, d)$, we need to also answer incrementally next-larger $(j, 1), \ldots$, next-larger $(j, d-1)$, taking overall $O(d)$ time.
} 


\section{Preliminaries}

Our results make use of a number of popular succinct data structures, which we list below for the sake of completeness.

\section{$2.1 \quad$ Bit-vectors}

A bit-vector $B[1, n]$ is an array of $n$ bits. We will be interested in solving two queries on it: $\operatorname{rank}_{b}(B, i)$ tells the number of occurrences of bit $b$ in $B[1, i]$, and $\operatorname{select}_{b}(B, j)$ gives the position of the $j$ th occurrence of bit $b$ in $B$. We will use the following result:

Lemma 1 ([10]) A bit-vector $B[1, n]$ can be stored in $n+o(n)$ bits (that is, o(n) bits on top of $B$ itself) so that queries rank and select are answered in $O(1)$ time.

When the number $m$ of $1 \mathrm{~s}$ in $B[1, n]$ is small, the following result will be of interest as well:

Lemma 2 ([31]) A bit-vector $B[1, n]$ with $m 1 s$ can be stored in $m \lg (n / m)+O(m)+o(n)$ bits, so that queries rank and select are answered in $O(1)$ time.

Note that from this compressed representation we can still retrieve any $B[i]=\operatorname{rank}_{1}(B, i)-$ $\operatorname{rank}_{1}(B, i-1)$ in constant time. If we aim at answering $\operatorname{rank}_{1}(B, i)$ only when $B[i]=1$, we can use less space, but now this is insufficient to recover the contents of $B$. The structure is called a monotone minimum perfect hash function (mmphf), as the rank values can be regarded as mapping elements in a universe $[1, n]$ to the domain $[1, m]$ while respecting the order:

Lemma 3 ([2]) Given a bit-vector $B[1, n]$ with $m 1 s$ we can answer queries rank $_{1}(B, i)$, whenever $B[i]=1$, in $O(1)$ time, using $O(m \lg \lg (n / m))$ bits, and without accessing $B$.

\subsection{Sequences}

A sequence $S[1, n]$ over alphabet $[1, \sigma]$ requires $n \lg \sigma$ bits if represented in plain form. Within almost the same space, we can answer not only the basic query access $(S, i)=S[i]$, but also the queries $\operatorname{rank}_{c}(S, i)$ and $\operatorname{select}_{c}(S, j)$ for any $c \in[1, \sigma]$, which are the natural extensions of the operations on bit-vectors:

Lemma 4 ([4, Thm. 6]) A sequence $S[1, n]$ over alphabet $[1, \sigma]$ can be stored in $n \lg \sigma+o(n \lg \sigma)$ bits, so that rank ${ }_{c}$ queries are solved in time $O\left(1+\lg \lg _{w} \sigma\right)$, select ${ }_{c}$ queries are solved in time $O(1)$, and access queries are answered in any time complexity of the form $\omega(1)$.

When the frequencies $n_{c}$ of the symbols $c \in[1, \sigma]$ are skewed, it is possible to use space close to the zeroth-order entropy of $S, n H_{0}(S)=\sum_{1 \leq c \leq \sigma} n_{c} \lg \left(n / n_{c}\right) \leq n \lg \sigma$ bits, and still answer the queries. For this article, the most useful result of this kind is the following:

Lemma 5 ([4, Thm. 7]) A sequence $S[1, n]$ over alphabet $[1, \sigma]$ can be stored in $n H_{0}(S)+o(n)$ bits, so that rank , select $_{c}$, and access queries are all solved in time $O\left(1+\lg _{w} \sigma\right)$.

To obtain constant-time access and select simultaneously when $\lg \sigma=\omega(w)$, we can resort to an earlier version of Lemma 4, which uses slightly more space: 
Lemma 6 ([19]) A sequence $S[1, n]$ over alphabet $[1, \sigma]$ can be stored in $(1+\epsilon) n \lg \sigma+o(n \lg \sigma)$ bits, for any constant $\epsilon>0$, so that rank $_{c}$ queries are solved in time $O(\lg \lg \sigma)$, select ${ }_{c}$ queries are solved in time $O(1)$, and access queries are answered in constant time $O(1 / \epsilon)$.

Finally, the following result gives a structure to support a restricted form of rank ${ }_{c}$ queries in constant time, by resorting to mmphfs.

Lemma 7 ([3, Sec. 3]) Given a sequence $S[1, n]$ over alphabet $[1, \sigma]$ we can answer queries rank $_{c}(S, i)$, where $S[i]=c$, in $O(1)$ time, using $O\left(n \lg H_{0}(S)\right)=O(n \lg \lg \sigma)$ bits, and without accessing $S$.

\subsection{Parentheses and trees}

A sequence $P[1,2 n]$ of parentheses '(' (opening) and ')' (closing) is balanced if, read left to right, there are never more closing than opening parentheses, and in total there is the same number of both. There is an opening parenthesis $P[j]$ matching each closing parenthesis $P[i]$ (this is the maximum $j<i$ such that $P[j, i]$ is also balanced). Such $j$ is found with operation findopen $(P, i)$, which will be used in this article. Concretely, we use the following result:

Lemma 8 ([26]) A balanced sequence of parentheses $P[1,2 n]$ can be stored in $2 n+o(n)$ bits (that is, o( $n)$ bits on top of $P$ itself) so that queries findopen $(\cdot)$ are answered in $O(1)$ time.

It is also useful to interpret $P$ as a bit-vector and add constant-time rank and select support, using $o(n)$ further bits (Lemma 1). The operations will be called rank), rank, select), and select $($.

A parenthesis sequence $P[1,2 n]$ can be used to represent a general ordinal tree of $n$ nodes, so that a large number of tree operations are supported in constant time. The next lemma lists those that will be used in this article:

Lemma 9 ([29]) An ordinal tree of $n$ nodes can be represented in $2 n+o(n)$ bits, so that the following operations are supported in constant time, among others: compute the parent of a node $v$, compute the ith child of a node $v$, find the mth left-to-right leaf, compute the preorder of a node $v$ and the node with preorder $r$, compute the depth of a node $v$, determine if a node $v$ is an ancestor of another node $u$, compute the ancestor at any distance $d$ of a node $v$, compute the subtree size of a node $v$, and find the internal node with inorder $s$ (leaves not counted).

We will use this lemma to represent binary trees where internal nodes always have two children. Then the left child of a node is the first and the right child is the second. Moreover, the inorder of an internal node is uniquely defined.

\section{$2.4 \quad$ Predecessor queries}

Given an increasing array $P[1, \kappa]$ of values in $[1, m]$, a predecessor query finds, given $x$, the maximum $i$ with $P[i] \leq x$. One can represent $P$ as $\kappa 1$ s on a bitvector $B[1, m]$, so that the predecessor of $x$ is select ${ }_{1}\left(\operatorname{rank}_{1}(B, x)\right)$. Using Lemma 2 to represent $B$, the space is $O(\kappa \lg (m / \kappa))+o(m)$ bits and the time is constant. It is not possible, however, to have constant time without the $o(m)$-bits term [30]. In our article we will make heavy use of a structure called the succinct SB-tree:

Lemma 10 ([21, Lem. 3.3]) If we have independent constant-time access to $P[1, \kappa]$, we can solve predecessor queries on $P$ in time $O(1+\lg \kappa / \lg \lg m)$ using $O(\kappa \lg \lg m)$ bits, plus a precomputed table of size $o(m)$ that depends only on $m$. 


\begin{tabular}{|l|l|l|l|l|l|l|l|l|}
\hline 3 & 1 & 2 & 4 & 6 & 5 & 7 & 8 & 9 \\
\hline 3 & 1 & 2 & 4 & 6 & 5 & 7 & 8 & 9 \\
\hline 3 & 1 & 2 & 4 & 6 & 5 & 7 & 8 & 9 \\
\hline
\end{tabular}

\begin{tabular}{|l|l|l|l|l|l|l|l|l|}
\hline 3 & 1 & 2 & 4 & 6 & 5 & 7 & 8 & 9 \\
\hline 3 & 1 & 2 & 4 & 6 & 5 & 7 & 8 & 9 \\
\hline 3 & 1 & 2 & 4 & 6 & 5 & 7 & 8 & 9 \\
\hline
\end{tabular}

Figure 1: Illustration of our example of how the successive queries sel $(1, j, 3)$ (in bold rectangles) spot the successive values of the permutations $\pi_{i}$ (grayed cells). The six snapshots of the queries are shown in columwinse order.

Note that the $o(m)$ bits are still present, but they do not depend on $P$, thus we will have many succinct SB-trees and a single $o(m)$-bits table for all. Though better times, like $O(\lg \lg \kappa)$, can be obtained with structures that use $O(\kappa \lg m)$ bits [30], our results are not affected by the slower time of succinct SB-trees, whereas their lower space usage turns out to be fundamental.

\section{Lower bounds}

In this section we show that, given $A[1, n]$ and $k$, any encoding answering queries sel $(1, j, k)$ or top $(1, j, k)$ needs at least (essentially) $n \lg k$ bits. Note that these queries are weaker as they consider the first $j$ positions of $A$ rather than a range of its positions. The technique is to encode about $n / k$ arbitrary permutations of $[k]$ in $A$, in a way that they can be retrieved with either of those queries. Thus the encodings cannot use less space that what is necessary to encode those arbitrary permutations, that is, rougly $n / k \times \lg _{2} k !=\Omega(n \lg k)$ bits.

Assume for simplicity that $n=\ell k$, for some integer $\ell$. Consider an array $A$ of length $n$, initialized to $A[j]=j$, for $1 \leq j \leq n$, and then re-order its elements as follows: take $\ell-1$ permutations $\pi_{i}$ on $[k], 0 \leq i<\ell-1$, and permute the elements in the subarray $A[i k+1,(i+1) k]$ according to permutation $\pi_{i}$, where $A[i k+j]=i k+\pi_{i}(j)$ for $0 \leq i<\ell-1$ and $1 \leq j \leq k$. Note that the last $k$ elements of $A$ are not reordered, as they do not encode any $\pi_{i}$. Also, for $0 \leq i_{1}<i_{2}<\ell-1$, the elements in the subarray for $i=i_{1}$ are all smaller than the elements in the subarray for $i=i_{2}$.

We now show how to reconstruct the $\ell-1$ permutations by performing $\operatorname{sel}(1, j, k)$ queries on the array $A$. The main idea is easy to grasp with an example.

Example. Assume we have permutations $\pi_{0}=\left(\begin{array}{lll}3 & 1 & 2\end{array}\right)$ and $\pi_{1}=\left(\begin{array}{lll}1 & 3 & 2\end{array}\right)$ where $k=3$. Figure 1 illustrates the process. Our array is $A[1,9]=\langle 3,1,2,4,6,5,7,8,9\rangle$, where $\pi_{0}$ is encoded in $A[1,3]$ and $\pi_{1}$ in $A[4,6]$ (with values shifted by $i k=3$ ). Then, sel $(1,3,3)=2$ tells us that the minimum among the first 3 elements in $\pi_{0}$ (i.e. the 3 rd largest element) is at $\pi_{0}(2)$, so $\pi_{0}(2)=1$. Next, sel $(1,4,3)=3$ tells us that the second minimum (2nd largest element) in $\pi_{0}$ is at $\pi_{0}(3)$, so $\pi_{0}(3)=2$, and thus $\pi_{0}(1)=3$. This is because $A[1,4]$ contains $A[4]$, which must be larger than all $A[1,3]$, and thus the 3rd largest element in $A[1,4]$ must be the 2nd largest element in $A[1,3]$. With $\operatorname{sel}(1,6,3)=4$ we discover that the $3 r d$ element in $\pi_{1}$ is at $\pi_{1}(1)$, so $\pi_{1}(1)=1$, and so on.

Now we formalize the process described in the example.

Lemma 11 The position of the $k$ th largest value in the prefix $A[1, i k+j-1]$ is the position of value $(i-1) k+j$, for any $1 \leq i<\ell$ and $1 \leq j \leq k$.

Proof. Since the values of $A$ were initially increasing and then we locally permuted the blocks of length $k$, it holds that, for each $1 \leq i<\ell, A[x]<A[y]$ for any $x \leq i k$ and $y>i k$. Then the values 
in $A[i k+1, i k+j-1]$ are the largest of $A[1, i k+j-1]$, and the values in $A[(i-1) k+1, i k]$ are the largest of $A[1, i k]$. Thus, the $k$ th largest value in $A[1, i k+j-1]$ is the $(k-j+1)$ th largest value in $A[1, i k]$. This value is also the $(k-j+1)$ th largest value in $A[(i-1) k+1, i k]$, or which is the same, the $j$ th smallest value in $A[(i-1) k+1, i k]$. Thus, by the definition of $A[(i-1) k+1$, $i k]$, it is the value $(i-1) k+j$.

Therefore, sel $(1, i k+j-1, k)$, the position of the $k$ th value in the prefix $A[1, i k+j-1]$, is equal to $(i-1) k+\pi_{i-1}^{-1}(j)$, which is the position of value $(i-1) k+j$. Then, any $\pi_{i-1}$ can be easily computed with the $k-1$ queries $\operatorname{sel}(1, i k+j-1, k)$ for $1 \leq j \leq k-1$.

Since representing $\ell-1$ arbitrary permutations on $[k]$ requires $\lg \left((k !)^{\ell-1}\right)=(\ell-1) \lg k !=$ $(n / k-1)(k \lg k-O(k))=(n-k) \lg k-O(n-k)=n \lg k-O(n+k \lg k)$ bits, any encoding able to answer all queries sel $(1, j, k)$ on $A$ needs also this number of bits.

The proof applies to top $(1, j, k)$ as well, since we can reconstruct the value sel $(1, i k+j-1, k)$ from top $(\cdot)$ queries: $\operatorname{sel}(1, i k+j-1, k)$ is the only element that disappears from the answer set when we move from top $(1, i k+j-1, k)$ to top $(1, i k+j, k)$. As we move, the element $A[i k+j]$ enters in the answer and the element that was the smallest (i.e., the $k$ th), which belongs to $A[(i-1) k+1, i k]$, leaves the answer set.

Theorem 1 Any encoding of an array $A[1, n]$ answering sel $(\cdot)$ or top $(\cdot)$ queries, even if restricted to ranges $A[1, j]$ and for a fixed $k$ value, requires at least $n \lg k-O(n+k \lg k)$ bits of space.

\section{General approach}

We describe Jørgensen and Larsen's "shallow cuttings" idea [23], and the way Chan and Wilkinson [9] take advantage of it. In general terms, our encoding for sel(.) queries will implement their solution in an encoding scenario. This poses, however, a number of challenges that will be dealt with in the subsequent sections; the plan is described at the end of this section. Table 1 gives the notation used throughout the article.

\subsection{Shallow cuttings}

Let $A[1, n]$ be a permutation on $[n]$. Consider each entry $A[i]$ as a point $(x, y)=(i, A[i])$, and set a parameter $\kappa$. A horizontal line sweeps the grid space $[1, n] \times[1, n]$ from $y=n$ (top) to $y=1$ (bottom). The points hit are included in a single root cell, which spans a three-sided area called a slab, of the form $[1, n] \times[y, n]$, which includes all the points of the cell. Once we reach a point $\left(x^{*}, y^{*}\right)$ that makes the root cell contain $2 \kappa$ points, we close the cell and leave its slab with its definitive area $[1, n] \times\left[y^{*}, n\right]$.

Let $x_{\text {split }}$ be the $\kappa$ th smallest $x$-coordinate in the above root cell. This is called the split point. The sweeping process is repeated recursively on each of the two grid spaces $\left[1, x_{\text {split }}\right] \times[1, n]$ and $\left[x_{\text {split }}+1, n\right] \times[1, n]$. This will create two children cells as follows. They will contain the topmost points whose $x$-coordinates are $\leq x_{\text {split }}$ and $>x_{\text {split }}$, respectively. Their slabs will grow downwards as we continue with the sweeping process, independently for each cell. When those cells, in turn, reach size $2 \kappa$, we close them, find their split points, and continue the recursion on the resulting grid spaces. The recursive process terminates on a final cell when less than $2 \kappa$ points are left in the current grid space. 


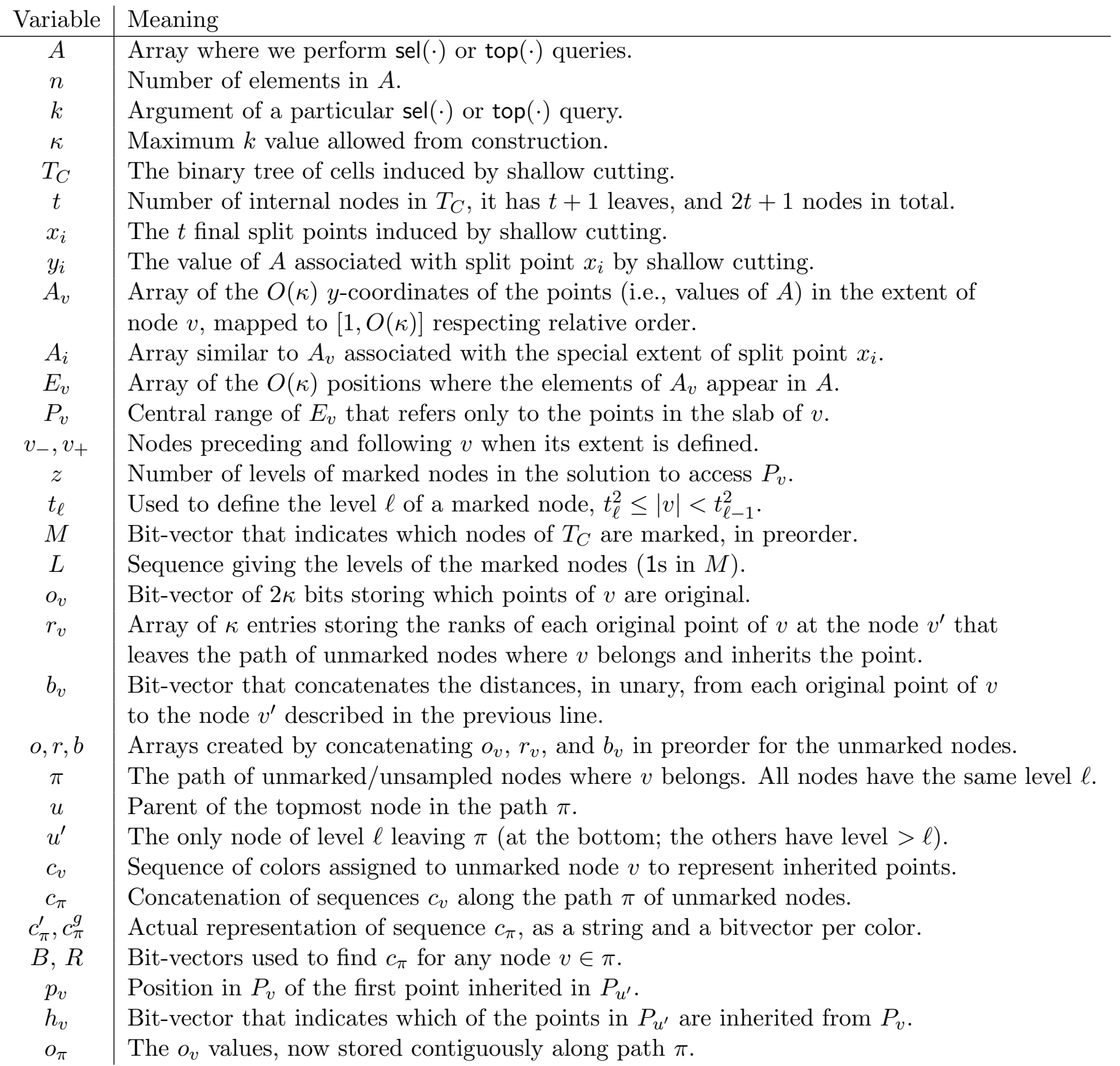

Table 1: Notation. 
A binary tree $T_{C}$ is created to reflect the cell refinement process (see Figure 2). The root cell is associated with the root node of $T_{C}$, the first two children cells to the left $\left(\left[1, x_{\text {split }}\right]\right)$ and right $\left(\left[x_{\text {split }}+1, n\right]\right)$ children of the root, and so on. The leaves of $T_{C}$ are associated with the final cells, which have not been split and contain $\kappa$ to $2 \kappa-1$ points (unless $n<\kappa$, in which case only a root cell exists).

At any moment of the sweeping process, we have a sequence of points $x_{1}<x_{2}<\ldots$ that have been chosen as split points; new points are inserted anywhere in the sequence as further cells are split. These points delimit the $x$-coordinate slab ranges of which are the leaves of $T_{C}$ at the current moment of the sweep. When the next split occurs, say within the slab covering interval $\left[x_{i}+1, x_{i+1}\right]$, we obtain two new cells, whose slabs cover the $x$-coordinate intervals $\left[x_{i}+1, x_{\text {split }}\right]$ and $\left[x_{\text {split }}+1, x_{i+1}\right]$. We associate the keys $\left[x_{i}+1, x_{\text {split }}\right]$ and $\left[x_{\text {split }}+1, x_{i+1}\right]$ and the extents $\left[x_{i-1}+1, x_{i+1}\right]$ and $\left[x_{i}+1, x_{i+2}\right]$, respectively, with the two new cells (assume further split points 0 and $n$ in the extremes).

When the sweep finishes, $T_{C}$ has $t$ internal nodes and $t+1$ leaves, and there are $t+2$ split points $0=x_{0}<x_{1}<x_{2}<\ldots<x_{t}<x_{t+1}=n$ (writing 0 and $n$ explicitly), which delimit the slabs of the final leaves of $T_{C}$. In the following, we will use $x_{i}$ to refer to these final split points. In addition to the extents associated with cells, we associate the special extents $\left[x_{i-1}+1, x_{i+1}\right]$ with the split points $x_{i}$, for $1 \leq i \leq t$. The root of $T_{C}$ has key and extent $[1, n]$. Note that, since leaves contain successive positions of $A$, it holds $\kappa \leq x_{i+1}-x_{i}<2 \kappa$ for all $i$ (if $n \geq \kappa$ ).

Example. Figure 2 gives an example (values $y_{i}$ will be defined soon). Note that the child slabs inherit half of the points of their parent slab.

This construction has a number of key properties [23]:

1. It creates $O(t)=O(n / \kappa)$ cells, each containing $\kappa$ to $2 \kappa$ points (if $n \geq \kappa$ ).

2. If $c$ is the cell of the highest (closest to the root) node $v \in T_{C}$ whose key is contained in a query range $A[i, j]$, then $[i, j]$ is contained in the extent of $c$.

3. The top- $\kappa$ values in $A[i, j]$ belong to the union of the 3 cells comprising the extent of $c$ (these contain at most $6 \kappa$ points).

\subsection{Optimal-time select queries}

Using the properties of shallow cuttings, Chan and Wilkinson [9] reduce the $O(\lg n / \lg \lg n)$ time of Brodal and Jørgensen [8] as follows. At each node $v \in T_{C}$, they store the structure of Brodal and Jørgensen for the array $A_{v}[1, O(\kappa)]$ of the $y$-coordinates of the points in the extent of $v$. Actually, they store in $A_{v}$ the local permutation in $[O(\kappa)]$ induced by the relative ordering in $A$, thus $A_{v}$ requires $O(\kappa \lg \kappa)$ bits in each $v$ and $O(n \lg \kappa)$ bits in total. The structure for range selection also uses $O(\kappa \lg \kappa)$ bits and answers queries in time $O(1+\lg \kappa / \lg \lg n) .{ }^{3}$ They also store an array $E_{v}[1, O(\kappa)]$, so that $E_{v}[i]$ is the position in $A[1, n]$ of the value stored in $A_{v}[i]$. For the special extents associated with split points $x_{i}$, they also store structures $A_{i}$ analogous to the structures $A_{v} .{ }^{4}$ The structures $A_{i}$ add up to $O(n \lg \kappa)$ bits, since they are built on sub-arrays of length up to $4 \kappa$ whose contents are mapped to the range $[1, O(\kappa)]$.

\footnotetext{
${ }^{3}$ One could expect time $O(1+\lg \kappa / \lg \lg \kappa)$, but the denominator may stay at $\lg \lg n$ by the use of global precomputed tables of total size $o(n)$.

${ }^{4}$ Arrays $E_{i}$ are not necessary because the special extents refer to contiguous ranges in $A$.
} 


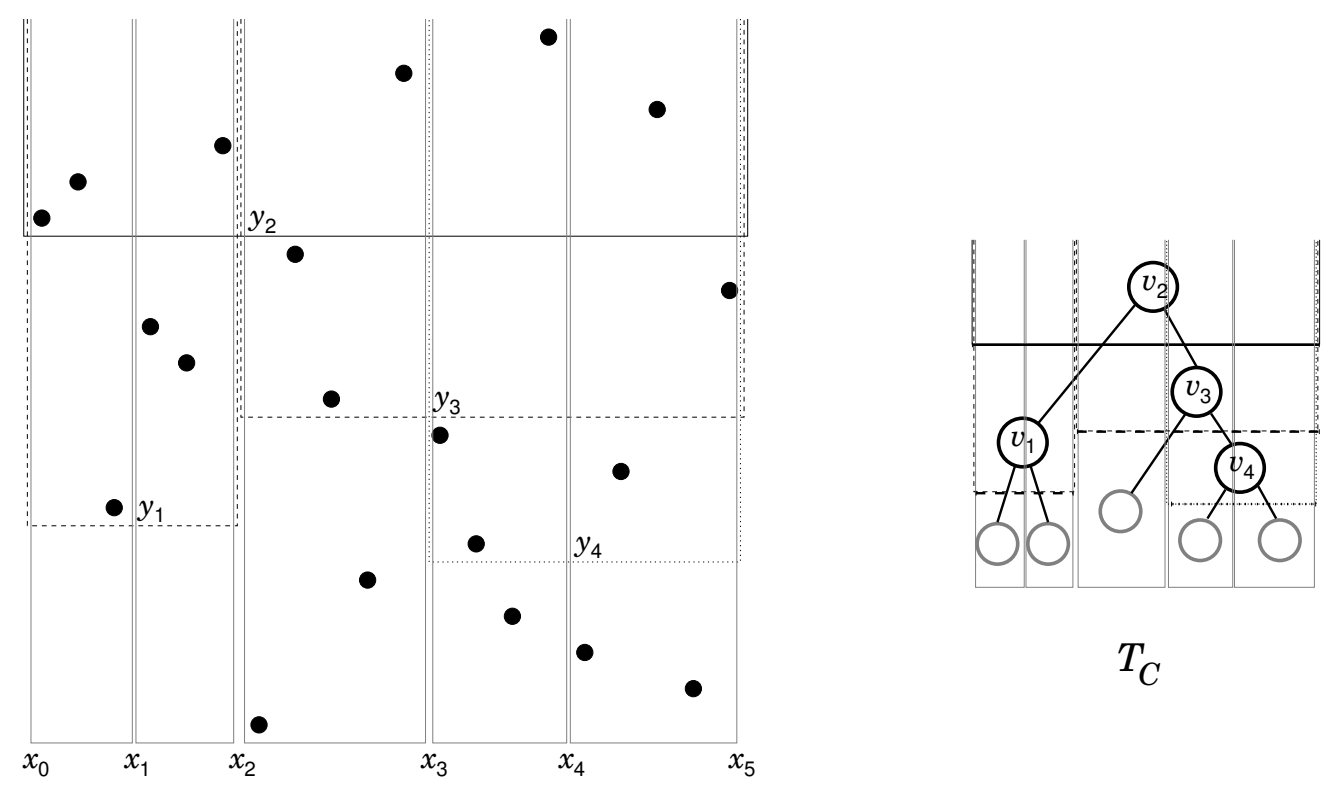

Figure 2: An example of the shallow cutting process with $\kappa=3$. On the left, the points are swept top to bottom. First, the root cell is closed when $x_{0}$ is found (the root slab is shown with a black solid line). The splitting point is $x_{2}$. Then its two children (slabs in black dashed lines) are formed. A third slab in black dotted lines is the right child of the right child of the root. The grayed lines show the slabs of the child cells. We show the split points $x_{i}$ and their associated $y^{*}$ values, $y_{i}$. On the right, the induced binary tree $T_{C}$ is shown, with leaves in gray. The keys of the internal nodes are the horzontal intervals shown in bold lines, and their identifiers are $v_{i}$, where $i$ is their inorder number.

Property 3 of shallow cuttings implies that the $k$ th largest element of $A[i, j]$, for any $k \leq \kappa$, is also the $k$ th largest value in $A_{v}[l, r]$, where $v$ is the node that corresponds to interval $A[i, j]$ by property 2 and $E_{v}[l-1]<i \leq j<E_{v}[r+1]$ are the elements in the extent of node $v$ enclosing $[i, j]$ most tightly. Thus query $\operatorname{sel}(i, j, k)$ on $A$ is mapped to query $p=\operatorname{sel}(l, r, k)$ on $A_{v}$. Once the local answer is found in $A_{v}[o]$, the global answer is $E_{v}[o]$.

Summing up, the main ingredients are based on the funtionalities of tree $T_{C}$, and arrays $E_{v}$, $A_{v}$ and $A_{i}$. Chan and Wilkinson [9] manage to store them in $O(n(\lg \kappa+\lg \lg n+(\lg n) / \kappa))$ bits, which gives $O(n \lg n)$ bits when added over a set of suitable $\kappa$ values (their structure works for every $1 \leq k \leq n$, so several $\kappa$-capped structures are built). Also, their solution requires to access $A$ and thus does not immediately translate into our setting.

\subsection{Encodings for optimal-time select queries}

Our general plan is to derive an encoding from the strategy of Chan and Wilkinson, which retains the optimal time for sel(.) but reduces the space to $O(n \lg \kappa)$ and does not access $A$. This requires addressing several challenges.

1. In Section 5 we design a succinct representation of $T_{C}$ that is able to find the node $v$ given the interval $A[i, j]$, so that from $v$ we gain access to the data associated with node $v$ in constant time. This structure uses $O((n / \kappa) \lg \kappa)+o(n)$ bits. Associated with each node $v$ we will store 
Chan and Wilkinson's structures $A_{v}$ for range selection (whose space is $O(\kappa \lg \kappa)$ bits and thus can be afforded), and a data structure that simulates array $E_{v}$ (as its direct representation cannot be afforded). We will also store the structures $A_{i}$ associated with the split points $x_{i}$.

2. In Section 6 we provide constant-time access to any $E_{v}$ using $O(n \lg \kappa)$ bits. Together with the previous result, this already yields an $O(\lg \kappa)$ time algorithm for sel $(\cdot)$ queries, as we can first find the node $v$ in constant time, then do a binary search for $l$ and $r$ in $E_{v}$, then run the range selection query on $A_{v}[l, r]$ in time $O(1+\lg \kappa / \lg \lg n)$, and finally return $E_{v}[o]$ in $O(1)$ time. Our representation of $E_{v}$ uses a hierarchical marking of nodes plus a color-based encoding of the inheritance of points along cells in paths of unmarked nodes in $T_{C}$.

3. In Section 7 we address the bottleneck of the previous solution: we replace the binary search by fast predecessor queries on $E_{v}$, so as to obtain $O(1+\lg \kappa / \lg \lg n)$ time. This is obtained by storing succinct SB-trees [21] on some sampled nodes (which include at least all the marked nodes), and searches on the inheritance information along paths of unsampled nodes, using global precomputed tables.

4. In Section 8 we wrap up the results in order to prove Theorem 2. Then we show how to answer top- $k$ queries by using an existing linear-space technique [6] on a reduced universe. This proves Theorem 3 .

Theorem 2 Given an array $A[1, n]$ and a value $\kappa$, there is an encoding of $A$ that uses $O(n \lg \kappa)$ bits and supports the query $\operatorname{sel}(i, j, k)$ in time $O(1+\lg k / \lg \lg n)$ for any $k \leq \kappa$.

Theorem 3 Given an array $A[1, n]$ and a value $\kappa$, there is an encoding of $A$ that uses $O(n \lg \kappa)$ bits and supports the query top $(i, j, k)$ in time $O(k)$, for any $k \leq \kappa$.

\section{Shallow cuttings in succinct space}

In this section we show how to represent the shallow cutting structure using $O((n / \kappa) \lg \kappa)+o(n)$ bits so that, given the query interval $[i, j]$, we obtain the corresponding node $v \in T_{C}$ according to property 2 of shallow cuttings, and then give access to the structures associated with node $v$. We will also need to find, given $v$, the two "neighbor" nodes $v_{-}$and $v_{+}$that define the extent of $v$, and map between nodes and their keys in both directions.

Finding the maximal range of split points. Our first structure is a bit-vector $S[0, n]$ that marks the split points $x_{i}$, that is, $S\left[x_{i}\right]=1$ for all $0 \leq i \leq t+1$ and $S[j]=0$ elsewhere. Since $S$ has only $t+2$ bits set out of $n$, we can represent it in compressed form (Lemma 2) so that it requires $t \lg (n / t)+O(t)+o(n)=O((n / \kappa) \lg \kappa)+o(n)$ bits of space and supports operations $\operatorname{rank}_{b}(S, i)$ and $\operatorname{select}_{b}(S, j)$ in constant time.

With this representation of $S$ we find in constant time the range $[m, M]$ of split points contained in $A[i, j]$. More precisely, we find the largest range $[m, M]$ such that $i \leq x_{m}<\ldots<x_{M} \leq j$, in constant time with $m=\operatorname{rank}_{1}(S, i-1)+1$ and $M=\operatorname{rank}_{1}(S, j)$. Note that the range $[m, M]$ can contain zero split points in some cases. We have the following result:

Lemma 12 Given the range $[i, j]$, we can find in $O(1)$ time the maximal range $[m, M]$ of split points (if any) contained in $[i, j]$ with a structure that uses $O((n / \kappa) \lg \kappa)+o(n)$ bits of space. 
If the range $[m, M]$ contains zero or one split points (i.e., $m \geq M$ ), then $[i, j]$ does not contain a complete cell: ${ }^{5}$ either $[i, j]$ is fully contained in the range of the $m$ th left-to-right leaf of $T_{C}$ (and contains no split points) or $[i, j]$ starts in the range of the $m$ th leaf and ends in that of the $(m+1)$ th leaf of $T_{C}$ (and contains one split point). In both situations, the range $[i, j]$ is contained in the special extent of the $m$ th split point of $T_{C},\left[x_{m-1}+1, x_{m+1}\right]$, recalling that $x_{m}$ is found using the bit-vector $S$. In this simple case, we compute the offset $o=\operatorname{select}_{1}(S, m-1)$, perform the query sel $(i-o, j-o, k)$ on the structure $A_{m}$ associated with split point $x_{m}$, and remap the answer to the global position by just adding $o$.

In the sequel we consider the more complex case of two or more split points, that is, $m<M$.

Finding the key of the node $v$ for a range $A[i, j]$. If $m<M$, the following procedure finds the desired key [23]. Find, within $x_{m}, \ldots, x_{M}$, the split point $x_{r}$ with maximum associated $y_{r^{-}}$ coordinate (this is the $y^{*}$ coordinate given to the slab of the cell that was closed when $x_{r}$ was chosen as a split point). Find the split point $x_{s}$ with the second maximum. If $s<r$ (i.e., $x_{s}$ is to the left of $x_{r}$ ), then the key of the desired node $v$ is $\left[x_{s}+1, x_{r}\right]$, otherwise it is $\left[x_{r}+1, x_{s}\right]$.

To find the first and second maxima, let the array $Y[1, t]=y_{1}, \ldots, y_{t}$ contain the $y^{*}$ values associated with the split points $x_{1}, \ldots, x_{t}$. We do not represent $Y$ itself, but rather store a range top-2 encoding of it [11]. This structure requires $O(t)=O(n / \kappa)$ bits and returns the positions of the first and second maxima in $Y[m, M], x_{r}$ and $x_{s}$, in $O(1)$ time. ${ }^{6}$ We have the following result:

Lemma 13 Given split points $x_{m}<x_{M}$, we can find in $O(1)$ time the maximal key of a node $v \in T_{C}$ that is contained in $\left[x_{m}+1, x_{M}\right]$, with a structure that uses $O(n / \kappa)$ bits of space.

Example. See Figure 2 again, and consider a range $A[i, j]$ that contains $x_{1}$ to $x_{4}$. Then $r=2$ and $s=3$, and the key is $\left[x_{2}+1, x_{3}\right]$, because $y_{2}=\max \left\{y_{1}, y_{2}, y_{3}, y_{4}\right\}$ and $y_{3}$ is the second maximum. Instead, if $A[i, j]$ contains $x_{3}$ to $x_{4}$, then $r=3$ and $s=4$ because $y_{3}>y_{4}$.

Finding the extent of $v$. Assume w.l.o.g. that $r<s$ and thus the desired key is $\left[x_{r}+1, x_{s}\right]$; the case $\left[x_{s}+1, x_{r}\right]$ is symmetric. To compute the extent of this key we need to find the split points that, at the moment when the key $\left[x_{r}+1, x_{s}\right]$ was created during the sweep, preceded $x_{r}$ and followed $x_{s}$. Let us call these split points $x_{r^{\prime}}$ and $x_{s^{\prime}}$, respectively. Here we use the encoding for prev-larger and next-larger queries described at the end of Section 1.

At the time we created the split point $x_{s}$, the split points that existed were precisely those with $y^{*}$ value larger than that associated with $x_{s}$. Thus, since $x_{r}<x_{s}$, the split point that followed $x_{s}$ is $x_{s^{\prime}}$, with $s^{\prime}=$ next-larger $(s, 1)$, the leftmost value in $Y[s+1, t+1]$ that is larger than $Y[s]$ (assume $Y[t+1]=n+1$ so this is always defined). Similarly, since all the values in $Y[r+1, s-1]$ are smaller than $Y[s]$, and $Y[r]>Y[s]$, the split point that preceded $x_{r}$ when $x_{s}$ was created was $x_{r^{\prime}}$, with $r^{\prime}=\operatorname{prev-larger}(s, 2)$, the second rightmost value in $Y[0, s-1]$ that is larger than $Y[s]$ (assume $Y[0]=n+1$ so this is always defined). ${ }^{7}$ In Section 5.1 we show how to support prev-larger and next-larger queries in constant time using $O(t)=O(n / \kappa)$ bits of space. Then the extent is $\left[x_{r^{\prime}}+1, x_{s^{\prime}}\right]$.

\footnotetext{
${ }^{5}$ In some border cases it can, but these are still correctly handled as indicated here.

${ }^{6}$ Note that it is not a matter of obtaining $r=\operatorname{rmq}(m, M)$ and then choosing $s$ from $s_{1}=\mathrm{rmq}(m, r-1)$ and $s_{2}=\operatorname{rmq}(r+1, M)$, since we have no way to compare $Y\left[s_{1}\right]$ with $Y\left[s_{2}\right]$ if we do not store $Y$.

${ }^{7}$ Note that prev-larger $(s, 2)$ is not necessarily prev-larger $(\operatorname{prev-larger}(s, 1), 1)=\operatorname{prev-larger}(r, 1)$ as there might be an element $x$ to the left of $Y[r]$ such that $Y[r]>x>Y[s]$.
} 
Lemma 14 Given the key of a node $v \in T_{C}$, and knowing which of its extremes has a lower $y$ value, we can obtain the extent of $v$ in $O(1)$ time with a structure that uses $O(n / \kappa)$ bits of space.

Example. In Figure 2, for the key $\left[x_{2}+1, x_{3}\right]$, we find the extent $\left[x_{0}+1, x_{5}\right]$, whereas for the key $\left[x_{3}+1, x_{4}\right]$, the extent is $\left[x_{2}+1, x_{5}\right]$. In both cases, the extent contains the range $A[i, j]$.

Finding the node with a given key. We have obtained the key of $v$, but not yet $v$. Similarly, we have obtained its extent, but not its corresponding neighboring nodes $v_{-}$and $v_{+}$. The structure $A_{v}$ contains the data corresponding to the extent of $v$, but we will also need to refer to its neighboring nodes in order to decode the results obtained in $A_{v}$.

To reference the nodes, we will represent the topology of $T_{C}$, which has $2 t+1$ nodes, with the succinct tree representation of Lemma 9. It uses $4 t+2=O(n / \kappa)$ bits of space and supports all the operations we need, in constant time.

If the key of node $v$ is $\left[x_{r}+1, x_{s}\right]$ and its extent is $\left[x_{r^{\prime}}+1, x_{s^{\prime}}\right]$, then the neighbor nodes of $v$ will be those with keys $\left[x_{r^{\prime}}+1, x_{r}\right]$ and $\left[x_{s}+1, x_{s^{\prime}}\right]$. In general, we will need to find the nodes corresponding to arbitrary keys.

Given a key $\left[x_{r}+1, x_{s}\right]$, where $Y[r]>Y[s]$, we can compute the corresponding node $v \in T_{C}$ as follows. Since this key was created with the split point $x_{s}$, the corresponding node of $T_{C}$ is the left child of the $s$-th node of $T_{C}$ in inorder [23]. This node with inorder $s$ is computed in constant time in our representation (Lemma 9), and then we can also compute its left child in constant time. If, instead, the key is $\left[x_{s}+1, x_{r}\right]$ (still with $Y[r]>Y[s]$ ), then $v$ is the right child of the $s$ th node in inorder.

Example. Again in Figure 2, the key $\left[x_{2}+1, x_{3}\right]$ holds $y_{2}>y_{3}$, thus we take the internal node of $T_{C}$ with inorder 3 (that is, $v_{3}$ ), and the desired node is its left child (that is, the third left-to-right leaf). Consider instead the key $\left[x_{3}, x_{5}\right]$. Since $y_{3}<y_{5}=n+1$, we take the internal node with inorder $3\left(v_{3}\right.$ again) and the answer is its right child, that is, $v_{4}$.

If the key is given but we do not know which is smaller between $Y[r]$ and $Y[s]$, we find the $r$ th inorder node $u_{r}$, the $s$ th inorder node $u_{s}$, and compare their depths in $T_{C}$; the deeper one corresponds to the smallest value. ${ }^{8}$ This is also useful to compute the extent of the resulting node, since the procedure we have given needs to know which of the two endpoints has a lower $y^{*}$ value.

Lemma 15 Given the key of a node $v \in T_{C}$, we can find the node $v$ itself, its extent, and its neighbor nodes $v_{-}$and $v_{+}$, in $O(1)$ time with a structure that uses $O(n / \kappa)$ bits of space.

Finding the key of a given node. Conversely, let $v$ be a node and assume we want to find its key $\left[x_{r}+1, x_{s}\right]$. If $v$ is the root, its key is $\left[x_{0}+1, x_{n}\right]$. Otherwise, we compute the parent node $u$ of $v$ in $T_{C}$, the inorder rank $i(u)$ of $u$, and the subtree size of $v,|v|$ (which is always odd since $T_{C}$ is binary). Then, if $v$ is the left child of $u$, we have $r=i(u)-(|v|+1) / 2$ and $s=i(u)$. If $v$ is a right child, then $r=i(u)$ and $s=i(u)+(|v|+1) / 2$. Recall that we can obtain the value $x_{i}=\operatorname{select}_{1}(S, i)$ of the $i$ th split point, for any $i$. The following lemma considers the space of the succinct representation of $T_{C}$ and the bitvector $S$.

\footnotetext{
${ }^{8}$ This only works if $\left[x_{r}+1, x_{s}\right]$ is a key; it cannot be used for the top- 2 problem we had mentioned.
} 
Lemma 16 Given a node $v \in T_{C}$, we can find its corresponding key in $O(1)$ time with a structure that uses $O((n / \kappa) \lg \kappa)+o(n)$ bits of space.

Example. Consider the node $v_{3}$ in Figure 2. Its parent is $v_{2}$. Since $v_{3}$ is a right child, the inorder of $v_{2}$ is 2 , and $\left|v_{3}\right|=5$, we have $r=2$ and $s=2+(5+1) / 2=5$. That is, the key of $v_{3}$ is $\left[x_{2}+1, x_{5}\right]$. Now consider the third left-to-right leaf. Its parent is $v_{3}$ (with inorder 3 ), the leaf is a left child and its subtree size is 1 . So we compute $r=3-(1+1) / 2=2$ and $s=3$, thus the key is $\left[x_{2}+1, x_{3}\right]$.

Associating structures with nodes. Once we have identified a node $v$, the succinct representation of $T_{C}$ yields its preorder rank $p(v)$ in constant time (Lemma 9). This is used to associate any desired data structure (such as $A_{v}$, for example) with the $p(v)$ th entry of an array.

\subsection{Computing next-larger and prev-larger queries}

We now show how to compute values next-larger $(j, d)$ and $\operatorname{prev-\operatorname {larger}}(j, d)$ for any $1 \leq j \leq t$ and $1 \leq d \leq \ell$, for some parameter $\ell$ given at construction time (see Section 1 for the definition of these queries). Our data structure will answer those queries in $O(d)$ time, using $O(\ell t)$ bits of space. For our needs, constant $\ell=2$ is sufficient, so the time is $O(1)$ and the space is $O(n / \kappa)$ bits.

We will describe the structure to support prev-larger queries for an array $Y[1, t]$; the one for next-larger is analogous. We define, for each element $Y[j], \ell$ pointers, $D_{1}[j] \ldots D_{\ell}[j]$, to the $\ell$ rightmost elements larger than $Y[j]$ that are in $Y[1, j-1]$.

Definition 1 Given an array $Y[1, t]$, we define arrays of pointers $D_{0}[1, t]$ to $D_{\ell}[1, t]$ as follows: $D_{0}[j]=j$, and $D_{d}[j]=\max \left(\left\{i<D_{d-1}[j]: Y[i]>Y[j]\right\} \cup\{0\}\right)$, for $d>0$.

We now prove a result that is essential for the space-efficient representation of all $D_{d}$ arrays, so that we can compute any prev-larger $(j, d)=D_{d}[j]$ in time $O(d)$. The following lemma shows that if we draw, for a given $d$, all the arcs starting at $D_{d-1}[j]$ and ending at $D_{d}[j]$ for all $j$, then no arcs "cross".

Lemma 17 Let $j_{1}, j_{2} \in[1, n]$ and $0<d \leq \ell$, and let us call $i_{1}=D_{d-1}\left[j_{1}\right]$ and $i_{2}=D_{d-1}\left[j_{2}\right]$. Then, if $i_{1}<i_{2}$ and $D_{d}\left[j_{2}\right]<i_{1}$, it holds $D_{d}\left[j_{1}\right] \geq D_{d}\left[j_{2}\right]$.

Proof. It must hold $Y\left[i_{1}\right]<Y\left[i_{2}\right]$, since otherwise $D_{d}\left[j_{2}\right] \geq i_{1}$ by Definition 1 (as it would hold $Y\left[j_{2}\right]<Y\left[i_{2}\right] \leq Y\left[i_{1}\right]$ and $\left.0<i_{1}<i_{2}\right)$, contradicting the hypothesis.

Now let us call $r_{1}=D_{d}\left[j_{1}\right]$ and $r_{2}=D_{d}\left[j_{2}\right]<i_{1}$. Then we have (1) $Y\left[r_{2}\right]>Y\left[j_{2}\right]$, because $r_{2}=D_{d}\left[j_{2}\right]$; (2) $Y\left[j_{2}\right] \geq Y\left[i_{1}\right]$, because otherwise it would be $r_{2}=D_{d}\left[j_{2}\right] \geq i_{1}$, as implied by Definition 1 (since $i_{1}<i_{2}=D_{d-1}\left[j_{2}\right]$ and $Y\left[i_{1}\right]>Y\left[j_{2}\right]$, and $r_{2} \geq i_{1}$ contradicts the hypothesis); and (3) $Y\left[i_{1}\right]>Y\left[j_{1}\right]$, because $i_{1}=D_{d-1}\left[j_{1}\right]$. Therefore, $Y\left[j_{1}\right]<Y\left[r_{2}\right]$, and then $r_{1}=D_{d}\left[j_{1}\right] \geq r_{2}$, as implied by Definition 1 since $r_{2}=D_{d}\left[j_{2}\right]<i_{1}=D_{d-1}\left[j_{1}\right]$.

Example. Figure 3 illustrates the lemma. The solid arcs cannot cross in the $x$ coordinate.

This property enables a space-efficient implementation of the pointers. We set bit-vector $T_{0}=1(10)^{t}$ to represent $D_{0}[j]=j$. We represent each "level" $d>0$ of pointers separately, as a set of arcs 


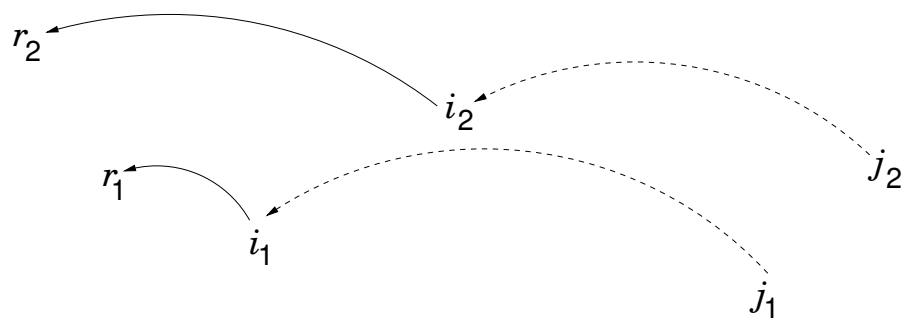

Figure 3: Illustration of Lemma 17, where $r_{1}=D_{d}\left[j_{1}\right]$ and $r_{2}=D_{d}\left[j_{2}\right]$. The heights of the elements represent their $y$ value (higher is larger). The dashed arrows represent $D_{d-1}$ and the solid arrows the step between $D_{d-1}$ and $D_{d}$.

leading from $D_{d-1}[j]$ to $D_{d}[j]$. For a level $d>0$ and for any $0 \leq i \leq t$, let $p_{d}[i]=\left|\left\{j, D_{d}[j]=i\right\}\right|$ be the number of pointers of level $d$ that point to position $i$. We then store a bit-vector

$$
T_{d}[1,2 t+1]=10^{p_{d}[0]} 10^{p_{d}[1]} 10^{p_{d}[2]} \ldots 10^{p_{d}[t]},
$$

where we mark the number of times each position is the target of pointers from level $d$. Each 1 corresponds to a new position and each 0 to the target of an arc. Note that the sources of those arcs correspond to the $0 \mathrm{~s}$ in bit-vector $T_{d-1}$, that is, to arcs that go from $D_{d-2}[j]$ to $D_{d-1}[j]$. Arcs that enter the same position $i$ are sorted according to their source position, so that we associate the leftmost $0 \mathrm{~s}$ of $0^{p_{d}}[i]$ with the arcs with the rightmost sources. Conversely, we associate the rightmost $0 \mathrm{~s}$ of $0^{p_{d-1}[i]}$ with the arcs with the leftmost targets. This rule ensures that those arcs entering, or leaving from, the same position do not cross in $T_{d}$ (as implied by Lemma 17). The matching between sources and targets is represented with a balanced sequence of parentheses (Lemma 8)

$$
B_{d}[1,2 t]=\left({ }^{p_{d-1}[0]}\right)^{p_{d-1}[0]}\left(p_{d}[0]-p_{d-1}[0]\right)^{p_{d-1}[1]}\left({ }^{p_{d}[1]}\right)^{p_{d-1}[2]}\left({ }^{p_{d}[2]} \ldots\right)^{p_{d-1}[t]}
$$

This sequence matches arc targets (opening parentheses) and sources (their corresponding closing parentheses). For example, take $T_{1}=101001001011011001011, T_{2}=100010010010110110111$, and $B_{2}=()(())(())(()()())()$ in Figure 4 (right column, on the center). Each 0 in $T_{2}$ represents a target corresponding to a parenthesis '(' in $B_{2}$, and it matches the 0 in $T_{1}$ that is the corresponding source represented by the companion ')' in $B_{2}$ : reading $B_{2}$ from left to right, the first 0 in $T_{2}$ is matched with the first 0 in $T_{1}$; the next two $0 \mathrm{~s}$ in $T_{2}$ are matched with the next two $0 \mathrm{~s}$ in $T_{1}$ by their nested pairs of parentheses, and so on. Here the enclosing pair of parentheses in $(()()())$ from $B_{2}$ matches the sixth 0 in $T_{2}$ with the ninth 0 in $T_{1}$ (see the corresponding arc $(7,2)$ in Figure 4 and observe that there are $7+1$ preceding $1 \mathrm{~s}$ in $T_{1}$ and $2+1$ preceding $1 \mathrm{~s}$ in $T_{2}$ ).

In general we write $p_{d}[i]$ parentheses '(' before the $p_{d-1}[i+1]$ parentheses ')', so the targets in position $i$ of $T_{d}$ can match all the corresponding sources that are to the right of position $i$ in $T_{d-1}$. Since the first $p_{d-1}[0]$ sources in $T_{d-1}$ are special as they have targets at position 0 in $T_{d}$ (i.e. they induce the only self-loops), we start preceding these sources with $p_{d-1}[0]$ targets from the $p_{d}[0]$ ones in $T_{d}$, and the remaining $p_{d}[0]-p_{d-1}[0]$ targets are written after these sources in $B_{d}$.

The tracking from $d=0$ to $d=\ell$ will proceed by computing values $z_{d}$, so that the value of interest corresponds to the $z_{d}$ th 0 in $T_{d}$. The following formula computes $z_{d}$ from $z_{d-1}$ :

$$
\left.z_{d}=\operatorname{findopen}\left(B_{d}, \text { select }\right)\left(B_{d}, z_{d-1}\right)\right) .
$$




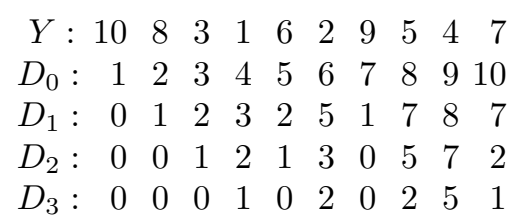

$T_{0}: 110101010101010101010$

$T_{1}: 101001001011011001011$

$T_{2}: 100010010010110110111$

$T_{3}: 100000100100111011111$

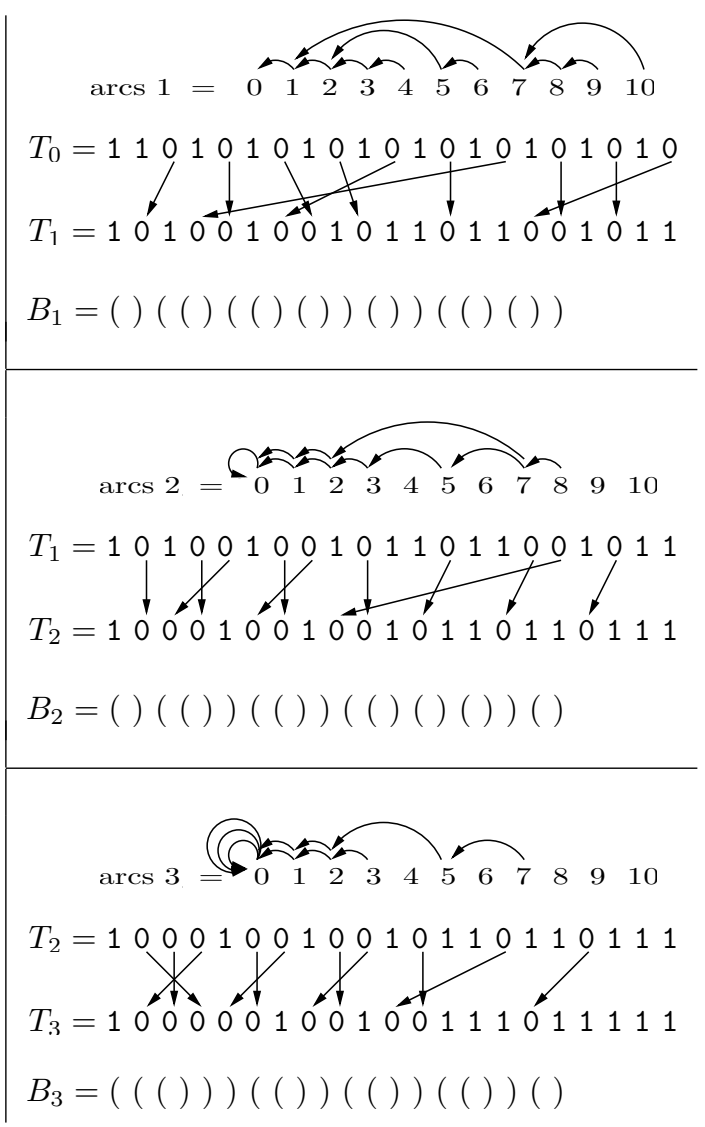

Figure 4: On the left, an example array $Y$, the data $D_{d}$ we store on it, and our representation, $T_{d}$ and $B_{d}$, of $D_{d}$. On the right, a graphical scheme of how the representation works.

We use the formula as follows. Starting with $z_{0}=j$, we use the formula up to $\ell$ times in order to find, consecutively, $z_{1}, z_{2}, \ldots, z_{\ell}$. At any point we have that $D_{d}[j]=\operatorname{rank}_{1}\left(T_{d}, \operatorname{select}_{0}\left(T_{d}, z_{d}\right)\right)-1=$ $\operatorname{select}_{0}\left(T_{d}, z_{d}\right)-z_{d}-1$. This gives the desired answer prev-larger $(j, d)=D_{d}[j]$. Note that we only store $T_{\ell}$ if we need to compute just $D_{\ell}[j]$.

Example. Figure 4 exemplifies the data structures on an array of $t=10$ elements and for $\ell=3$. On the left we show $Y$, the arrays $D_{0}$ to $D_{3}$, and their representation as the bit-vector $T_{d}$ and the parenthesis sequences $B_{d}$. On the right we show how the data structures work. For each $d$, we first draw the arcs that go from $D_{d-1}[j]$ to $D_{d}[j]$ (note that, for $d>1$, their sources may be far away from position $j$ ). If we want to compute $D_{d}[j]$, we start with the arc that leaves from $j$ at "arcs 1 ", and this gives us $D_{1}[j]$. Then we go to "arcs 2 " and find the arc that leaves from $D_{1}[j]$, which gives us $D_{2}[j]$. This way we compute any $D_{d}[j]$ in time $O(d)$. Note that, when several arcs lead to the same position, we are careful to pick the correct one in the next level. If the arrow arrives at position $i$ left to right, it corresponds in the next level to the $i$ th arrow that leaves, right to left. This is taken care of by the parentheses.

By adding select support to $T_{d}$, and select and findopen support to $B_{d}$ (Lemmas 1 and 8), we have that the overall space is $4 \ell t+o(\ell t)$ bits.

Theorem 4 Given an array $Y[1, t]$ and a parameter $\ell$, we can build a structure that uses $4 \ell t+o(\ell t)$ 
bits and answers any query prev-larger $(j, d)$ on $Y$ in time $O(d)$, for any $1 \leq i \leq t$ and $1 \leq d \leq \ell$, without accessing $Y$. If we only want to compute prev-larger $(j, \ell)$, the structure uses $2(\ell+1) t+o(\ell t)$ bits of space. An analogous structure solves queries next-larger $(j, d)$.

\section{Constant-time access to $E_{v}$}

In this section we describe a data structure that gives constant-time access to the values $E_{v}[1, O(\kappa)]$ in any node $v$. We recall the general picture for the sake of presentation. We begin with the input array $A[1, n]$, and create the tree $T_{C}$ of $2 t+1$ nodes for the shallow cuttings on the set of points $\{(i, A[i]) \mid 1 \leq i \leq n\}$ : note that each point is a pair given by a position $i$ (the $x$-coordinate) and its value $A[i]$ (the $y$-coordinate), and there is a one-to-one mapping between points and positions. Without introducing ambiguity, we thus refer to the points represented in $T_{C}$ for the properties but we actually store just their positions (not their values).

Specifically, consider a node $v \in T_{C}: E_{v}$ is the set of positions such that $E_{v}[i]$ is the position in $A$ of the value stored in $A_{v}[i]$, where array $A_{v}$ stores the values belonging to the extent of $v$ (essentially, letting $j=E_{v}[i]$, it is $A[j]=A_{v}[i]$, however the values in $A_{v}$ are mapped to the interval $[1, \mathcal{O}(\kappa)]$ respecting the order between values.) For any node $v \in T_{C}$, we want to encode $E_{v}$ so that each $E_{v}[i]$ can be retrieved in constant time. To this end, recall that $v_{-}$and $v_{+}$are the nodes that precede and follow $v$ in its extent: they can be accessed as shown in Lemma 15. Introducing the notation $P_{x}$ to indicate the subset of $O(\kappa)$ positions from $E_{v}$ whose corresponding points occur in the slab of node $x \in\left\{v_{-}, v, v_{+}\right\}$, we have that $E_{v}=P_{v_{-}}: P_{v}: P_{v_{+}}$. Hence, we will focus only on $P_{v}$ without loss of generality, as we can easily simulate the concatenation $E_{v}=P_{v_{-}}: P_{v}: P_{v_{+}}$. Concretely, in this section we prove the following result.

Theorem 5 Given the structures for constant-time navigation in $T_{C}$ (Lemma 9) and for handling shallow cuttings in $T_{C}$ (Lemmas 13 to 16), for any node $v \in T_{C}$, any position $P_{v}[i]$ can be retrieved in $O(1)$ time, with structures that use $O(n \lg \kappa)$ bits of space.

The main idea is that most nodes in $T_{C}$ cover a small span in $A$, and thus the $x$-coordinates of their points can be specified with a small offset. Nodes will be classified by subtree size, so that fewer bits are used for the $P_{v}$ arrays of lower nodes. Some nodes of each class of subtree sizes will be marked and all their points will be stored explicitly using this technique. For the unmarked nodes, we observe that the points in their cells are inherited by their descendants, so we will find a way to describe the (marked) descendant where each point is to be retrieved.

\subsection{Marking nodes}

We define an exponentially decreasing sequence of sizes as follows: $t_{0}=t$ and $t_{\ell+1}=\left\lceil\lg t_{\ell}\right\rceil$, until reaching a step $z$ such that $t_{z}=1$. Node $v$ will be of level $\ell$ if $t_{\ell}^{2} \leq|v|<t_{\ell-1}^{2}$ (recall that $|v|$ is the number of nodes in the subtree of $v$ ). For any $\ell \geq 1$, we mark a node $v \in T_{C}$ if it is of level $\ell$ and:

C1. it is a leaf or both its children are of level $>\ell$; or

C2. both its children are of level $\ell$; or

C3. it is the root or its parent is of level $<\ell$.

Note that in fact there are no nodes of level $\ell=0$. More generally, we have the following limit. 
Lemma 18 The number of marked nodes of level $\ell$ is $O\left(t / t_{\ell}^{2}\right)$.

Proof. The key property is that the descendants of $v$ are of the same level of $v$ or more. So nodes marked by $\mathrm{C} 1$ above cannot descend from each other, thus each such marked node has at least $t_{\ell}^{2}$ descendants not shared with another. As $T_{C}$ has $2 t+1$ nodes, there cannot be more than $(2 t+1) / t_{\ell}^{2}$ nodes marked by this condition. By the same key property, nodes marked by $\mathrm{C} 2$ form a binary tree whose leaves are those marked by $\mathrm{C} 1$, thus there are at most other $(2 t+1) / t_{\ell}^{2}$ nodes marked by $\mathrm{C} 2$. For $\mathrm{C} 3$, note that all unmarked nodes of level $\ell$ are in disjoint paths (otherwise the parent of two nodes of level $\ell$ would be marked by $\mathrm{C} 2$ ), and the path terminates in a node already marked by $\mathrm{C} 1$ or $\mathrm{C} 2$ (contrarily, a node of level $\ell$ marked by $\mathrm{C} 3$ must be a child of a node of level $<\ell$, and thus cannot descend from nodes of level $\ell$, by the key property). Therefore, C3 marks the highest node of each such isolated path leading to a node marked by $\mathrm{C} 1$ or $\mathrm{C} 2$, and thus the number of nodes marked this way is limited by those marked by $\mathrm{C} 1$ or $\mathrm{C} 2$.

\subsection{Handling marked nodes}

Marked nodes, across all the levels, are few enough to admit an essentially naive storage of their array $P_{v}$. If a marked node $v$ represents a slab with left boundary $x_{l}+1$, we store all its $P_{v}[o]$ values as the integers $P_{v}[o]-x_{l}$. As shown in Lemmas 13 and 15 , we know both $v$ and $x_{l}$, and thus we obtain $P_{v}[o]$ in constant time. Since a node of level $\ell$ contains less than $t_{\ell-1}^{2}$ descendants (about half of which are leaves), its slab spans less than $\left(t_{\ell-1}^{2}+1\right) / 2$ consecutive split points $x_{i}$, and thus less than $\kappa\left(t_{\ell-1}^{2}+1\right)$ positions in $A$. Thus, each such integer $P_{v}[o]-x_{l}$ can be represented using $\lg \left(\kappa\left(t_{\ell-1}^{2}+1\right)\right)=O\left(t_{\ell}+\lg \kappa\right)$ bits.

We need a few further structures to give constant-time access to structures $P_{v}$, since their size depend on the level of the node. Our succinct representation of $T_{C}$ gives the preorder rank $p(v)$ of node $v$ in constant time (Lemma 9 ). We store a bit-vector $M[1,2 t+1]$ where $M[p(v)]=1$ iff node $v$ is marked. Further, we store a string $L[1, O(t)]$ where we write down the level of each marked node, that is, $L\left[\operatorname{rank}_{1}(M, p(v))\right]=\ell$ iff $v$ is marked and of level $\ell$. Since every $\ell \leq \lg ^{*} t$, the alphabet of $L$ is $\left[0, \lg ^{*} t\right]$. Then we can represent $L$ using $|L| H_{0}(L)+o(t)$ bits so that operations access, rank, and select on $L$ take $O\left(1+\lg _{w} \lg ^{*} t\right)=O(1)$ time (Lemma 5$)$.

With $M$ and $L$ we can create separate storage areas per level for the explicit arrays $P_{v}$ of marked nodes, each of which uses the same space, $\kappa \lg \left(\kappa\left(t_{\ell-1}^{2}+1\right)\right)$ bits, for nodes of the same level $\ell$. If a node $v$ is marked (i.e., $M[p(v)]=1$ ) and is of level $\ell=L\left[\operatorname{rank}_{1}(M, p(v))\right]$, then we store its array $P_{v}$ as the $r$ th one in a separate sequence for level $\ell$, where $r=\operatorname{rank}_{\ell}\left(L, \operatorname{rank}_{1}(M, p(v))\right)$.

Lemma 19 Constant-time access to any entry in $P_{v}$ for any marked node $v$ can be provided within $O(n \lg \kappa)$ total bits of space.

Proof. We have explained how to store the arrays classified by level so as to provide constant-time access to any $P_{v}$. Let us now consider the space.

The arrays $P_{v}$ themselves use $O\left(\kappa\left(t_{\ell}+\lg \kappa\right)\right)$ bits each. The second term, $O(\kappa \lg \kappa)$ bits per marked node, adds up to $O(n \lg \kappa)$ bits overall. Since, by Lemma 18, there are $O\left(t / t_{\ell}^{2}\right)$ marked nodes of level $\ell$, the first term, $O\left(\kappa t_{\ell}\right)$, adds up to $O\left(\left(t / t_{\ell}^{2}\right) \cdot\left(\kappa t_{\ell}\right)\right)=O\left(n / t_{\ell}\right)$ bits over all the marked nodes of level $\ell$. Adding over all the levels $\ell$ we have $O(n) \sum_{\ell=0}^{z} 1 / t_{\ell}$. Since $t_{z}=1$ and $t_{\ell-1}>2^{t_{\ell}-1}$, it holds that $t_{z-s}>2^{s}$ for $s \geq 4$, and thus $\sum_{\ell=0}^{z} 1 / t_{\ell} \leq O(1)+\sum_{s \geq 4} 1 / 2^{s}=O(1)$, therefore the terms $O\left(\kappa t_{\ell}\right)$ add up to $O(n)$ bits. 
Bit-vector $M$ uses $2 t+1=O(n / \kappa)$ bits, whereas the storage of $L$ uses $|L| H_{0}(L)+o(t)$ bits. Letting $n_{\ell}$ be the number of occurrences of $\ell$ in $L$, we have $|L| H_{0}(L)=\sum_{\ell} n_{\ell} \lg \left(|L| / n_{\ell}\right)$. Since $n_{\ell} \lg \left(|L| / n_{\ell}\right)$ is increasing ${ }^{9}$ with $n_{\ell}$ and $n_{\ell}=O\left(t / t_{\ell}^{2}\right)$ by Lemma 18 , we have $|L| H_{0}(L) \leq$ $O(t) \sum_{\ell} \lg \left(t_{\ell}^{2}\right) / t_{\ell}^{2}=O(t) \sum_{\ell} \lg \left(t_{\ell}\right) / t_{\ell}^{2} \leq O(t) \sum_{\ell} 1 / t_{\ell}=O(t)$ (we showed in the previous paragraph that the sum is $O(1))$.

\subsection{Handling unmarked nodes}

The problem of supporting constant-time access to $P_{v}$ is solved for marked nodes, but $T_{C}$ may have $\Theta(t)$ unmarked nodes. To deal with unmarked nodes, we first observe that an unmarked node $v$ at level $\ell$ has exactly one level $\ell$ child and one child $x$ at level $>\ell$ (otherwise $v$ would be marked by C1 or C2). Furthermore, $x$ is marked by C3. Finally, the marked parent of an unmarked level $\ell$ node must be the root or at level $\ell$ itself. Thus, as already observed in the proof of Lemma 18, level- $\ell$ unmarked nodes form disjoint paths in $T_{C}$, and all the nodes adjacent to such paths are marked.

Now consider the points in slabs corresponding to unmarked nodes. When a cell is closed and split into two, the leftmost (rightmost) $\kappa$ points in its slab become part of its left (right) child cell. Thus, each child cell starts out with $\kappa$ inherited points, which are in common with its parent slab, and (at most) $\kappa$ further original points will be added to it before it is itself closed (becoming a child slab) and split.

For each point of node $v$, in $x$-coordinate order, we use a bit to specify if the point is inherited (0) or original (1). Let $o_{v}[1,2 \kappa]$ be this bit-vector, which will be stored for all the unmarked nodes $v \in T_{C}$, at a total cost of $O(n)$ bits. We now describe how to recover the position (contained in $\left.P_{v}\right)$ of an original and an inherited point, with different mechanisms.

\subsubsection{Retrieving the positions of original points}

Let $\pi$ be a path of unmarked nodes of level $\ell$, and let $v$ be an unmarked node in $\pi$. Each original point $p$ of $v$ must become an inherited point of some marked descendant $v^{\prime}$ that is adjacent to $\pi$ (recall that $v^{\prime}$ represents all the positions of its points explicitly). Thus the coordinate of each such original point $p$ can be specified by recording which marked descendant $v^{\prime}$ contains it, and the rank of $p$ among the points of $v^{\prime}$.

The ranks are stored in an array $r_{v}[1, \kappa]$, with one entry per original point in $v$. The distances require a more sophisticated mechanism. Suppose that the $j$ th original point in $v$ is in $v$ 's marked descendant $v^{\prime}$ at distance $d_{j}$ along $\pi$. Note that the point is inherited by the $d_{j}$ intermediate descendants of $v$ as well. Then we write the bit-vector $b_{v}=1^{d_{1}-1} 01^{d_{2}-1} 0 \ldots 1^{d_{\kappa}-1} 0$.

The vectors $o_{v}, r_{v}$ and $b_{v}$ are concatenated in the same preorder as the nodes. While vectors $o_{v}$ and $r_{v}$ are of fixed size, vectors $b_{v}$ are not. So we can concatenate all the bit-vectors $o_{v}[1,2 \kappa]$ and vectors $r_{v}[1, \kappa]$ in preorder into a global bit-vector $o[1, O(\kappa t)]=o[1, O(n)]$ and a global array $r[1, O(\kappa t)]=r[1, O(n)]$. Then, if $v$ is unmarked (i.e., $M[p(v)]=0), o_{v}[i]$ is at $o[2 \kappa(m-1)+i]$, where $m=\operatorname{rank}_{0}(M, p(v))$, and moreover $\operatorname{rank}_{1}\left(o_{v}, i\right)=\operatorname{rank}_{1}(o, 2 \kappa(m-1)+i)-\operatorname{rank}_{1}(o, 2 \kappa(m-1))$. Given any original point $o_{v}[i]=1$, it is the $j$ th original point for $j=\operatorname{rank}_{1}\left(o_{v}, i\right)$, and thus its corresponding entry is $r_{v}[j]$, which is found at $r\left[\operatorname{rank}_{1}(o, i)\right]$. Finally, we concatenate all the bit-vectors $b_{v}$ for the unmarked nodes $v$ in preorder creating a bit-vector $b$. If $o_{v}[i]=1$ and $j=\operatorname{rank}_{1}\left(o_{v}, i\right)$, then we

\footnotetext{
${ }^{9}$ At least for $n_{\ell} \leq|L| / e$. When $n_{\ell}$ is larger we can simply bound $n_{\ell} \lg \left(|L| / n_{\ell}\right)=O\left(n_{\ell}\right)$, thus we can remove all those large $n_{\ell}$ terms from the sum and add an extra term $O(t)$ to absorb them all.
} 
recover $d_{j}=\operatorname{select}_{0}\left(b_{v}, j\right)-$ select $_{0}\left(b_{v}, j-1\right)$. On the concatenated bit-vectors, for the original point $o[i]$ we compute $j=\operatorname{rank}_{1}(o, i)$ and then $d_{j}=\operatorname{select}_{0}(b, j)-\operatorname{select}_{0}(b, j-1)$.

Thus, to obtain the position $P_{v}[i]$ of an original point (i.e., with $o_{v}[i]=1$ ) in an unmarked node $v$, we obtain the distance $d_{j}$ to the marked descendant $v^{\prime}$ where $P_{v}[i]$ is stored. Since $v^{\prime}$ is marked, the answer obtained in constant time (Lemma 19) from $P_{v^{\prime}}\left[r\left[\operatorname{rank}_{1}(o, i)\right]\right]$ (to which we add the starting position of the slab of $v^{\prime}$ ).

The remaining problem is then to find the marked node $v^{\prime}$ leaving $\pi$ at distance $d_{j}$ from $v$. The strategy is to find the node $u^{\prime}$ that is "at the end" of $\pi$. More precisely, $u^{\prime}$ is a child of the lowest node of $\pi$ and is the only node leaving $\pi$ that is of the same level $\ell$ of $v$ (thus $u^{\prime}$ is marked). Since we can compute node depths and ancestors at any distance in constant time on $T_{C}$ (Lemma 9), we can compute the ancestor $a$ of $u^{\prime}$ that is at depth $\operatorname{depth}(v)+d_{j}-1$, and find $v^{\prime}$ as the child of $a$ that is not in $\pi$, that is, is not an ancestor of $u^{\prime}$.

There is a slight ambiguity to describe $v^{\prime}$ using $d_{j}$ : both $u^{\prime}$ and its sibling leave $\pi$, and they are at the same distance to their ancestors. To distinguish them, we encode $d_{j}+1$ instead of $d_{j}$ in $b_{v}$ to denote the node $u^{\prime}$, whereas its sibling is denoted with $d_{j}$ as usual. Therefore, when we compute $a$ and it holds $a=u^{\prime}$, we know that $v^{\prime}=u^{\prime}$.

We still need to find $u^{\prime}$. The key property is that $u^{\prime}$ is the highest marked node of level $\ell$ in the subtree of $v$. We calculate the subtree size of $v$ in constant time (Lemma 9) and hence its level $\ell{ }^{10}$ If the nodes are arranged in preorder, $u^{\prime}$ is the first node appearing after $p(v)$, $p\left(u^{\prime}\right)>p(v)$, which is marked $\left(M\left[p\left(u^{\prime}\right)\right]=1\right)$ and whose level is $L\left[\operatorname{rank}_{1}\left(M, p\left(u^{\prime}\right)\right)\right]=\ell$. This corresponds to the first occurrence of $\ell$ in $L$ after position $\operatorname{rank}_{1}(M, p(v))$, and is found in constant time with $p=\operatorname{select}_{\ell}\left(L, \operatorname{rank}_{\ell}\left(L, \operatorname{rank}_{1}(M, p(v))\right)+1\right)$. Then $p\left(u^{\prime}\right)$ is $\operatorname{select}_{1}(M, p)$. Finally, the tree representation gives us $u^{\prime}$ from its preorder rank $p\left(u^{\prime}\right)$ in constant time as well (Lemma 9).

Lemma 20 Constant-time access to the position $P_{v}[i]$ of any original point in the unmarked nodes $v$ can be provided within $O(n \lg \kappa)$ bits of space.

Proof. We have already explained how constant-time access is provided. Let us analyze the space. The arrays $o$ and $r$ require $O(n)$ and $O(n \lg \kappa)$ bits, respectively. To bound the space of array $b$ we claim that, summed across all the nodes $v$ in the path $\pi$, the arrays $b_{v}$ add up to $2|\pi| \kappa$ bits: each $b_{v}$ has $\kappa$ 0-bits, and each 1-bit in $b_{v}$ represents the same point when it is inherited in a descendant of $v$ along $\pi$. Since $\pi$ contains in total $|\pi| \kappa$ inherited points, the 1 s in all the bit-vectors $b_{v}$ of $\pi$ also add up to $|\pi| \kappa$. Thus, $|b|=\sum_{v \in T_{C}}\left|b_{v}\right|=O(t \kappa)=O(n)$ bits. Arrays $M$ and $L$ were already considered in Lemma 19.

\subsubsection{Retrieving the positions of inherited points}

We cannot use bit-vectors analogous to $b_{v}$ for the inherited points in $v$, as we cannot bound their size (because the same points are inherited over and over along $\pi$ ). For each inherited point $p$ in $v$, we instead specify which ancestor of $v$ on $\pi$ has $p$ as an original point, and then retrieve the position of the point as that of an original point in the ancestor using Lemma 20. If the ancestor is outside $\pi$, we specify the marked parent $u$ of the topmost unmarked node in $\pi$, and retrieve the

\footnotetext{
${ }^{10}$ To find the level in constant time from the subtree size, we can check directly for the case $\ell=0$, and store the other answers in a small table of $\lg ^{2} t$ cells.
} 
position from $P_{u}$ using Lemma 19 (as $u$ is marked). In the rest of this subsection, we assume that the ancestor is inside $\pi$.

To specify the ancestors, we code the points using $4 \kappa$ colors. Of these colors, $2 \kappa$ are said to be original colors and $2 \kappa$ are said to be inherited colors. For each original color $g$ there is a corresponding inherited color $g^{\prime}$. All the points in $u$ are given arbitrary distinct original colors. Then we traverse the nodes $v$ in $\pi$ top to bottom. If point $p$ in $v$ is inherited (from its parent $v^{\prime}$ ), we look at the color of $p$ in $v^{\prime}$. If $p$ has an original color $g$ in $v^{\prime}$, we give $p$ color $g^{\prime}$ in $v$. Otherwise, if $p$ is also inherited in $v^{\prime}$, having color $g^{\prime}$, it will also have color $g^{\prime}$ in $v$. On the other hand, if point $p$ is original in $v$, we give it one of the currently unused original colors: any color $g$ such that $g$ is not already an original color in $v$ and $g^{\prime}$ is not among the $\kappa$ inherited colors of $v$ can be used as the original color for $p$. Note that no colors $g$ and $g^{\prime}$ can be present simultaneously in any $v^{\prime}$, thus writing $g^{\prime}$ in $v$ unambiguously determines which color is inherited from $v^{\prime}$. The colors of node $v$ are represented in a string $c_{v}[1,2 \kappa]$, adding up to $O(n \lg \kappa)$ bits.

This scheme gives sufficient information to track the inheritance of points across $\pi$ : conceptually when a new, original, point $p$ appears in $v$, it is given an original color $g$. Then the point is inherited along the descendants of $v$ as long as color $g^{\prime}$ exists below $v$. Thus, to find the appropriate ancestor of $v$ that contains, as an original point, a given inherited point $p$ of color $g^{\prime}$, we concatenate all the color strings $c_{v}$ on $\pi$ into a string $c_{\pi}$, top to bottom, and ask for the nearest preceding occurrence of color $g$. Inside $c_{\pi}$, the subarray $c_{v}$ starts at position $2 \kappa(\operatorname{depth}(v)-\operatorname{depth}(u))+1$. Thus, we seek to find the rightmost $c_{\pi}[j]=g$ preceding some $c_{\pi}[i]=g^{\prime}$. With $j$, we have that $v^{\prime}$ is the ancestor of $v$ at depth $\operatorname{depth}(u)+\lceil j /(2 \kappa)\rceil-1$, and the position of the desired (original) point is $P_{v^{\prime}}[j \bmod 2 \kappa]$.

The sequence of colors $c_{\pi}$ will be associated with the last node $u^{\prime}$ of $\pi$, and all of them will be concatenated in preorder of those nodes $u^{\prime}$. A bit-vector $B[1, O(t)]$ will mark the starting position of each sequence $c_{\pi}$ in the concatenation (by chunks of $2 \kappa$ entries), and another bit-vector $R[1,2 t+1]$ contains all 0s except $R\left[p\left(u^{\prime}\right)\right]=1$ for all the nodes $u^{\prime}$ of all the paths $\pi$. Thus we have access to any individual sequence $c_{\pi}$ : for any $v \in \pi$ terminated in $u^{\prime}$ (Section 6.3.1 explains how to compute $\left.u^{\prime}\right), c_{\pi}$ starts at position $1+2 \kappa\left(\operatorname{select}_{1}\left(B, \operatorname{rank}_{1}\left(R, p\left(u^{\prime}\right)\right)\right)-1\right)$ of the concatenated sequence.

To find $j$, we will not represent $c_{\pi}$ directly, but rather $c_{\pi}^{\prime}$, where both the original colors $g$ and the inherited colors $g^{\prime}$ are written as $g$. To distinguish them, we store $2 \kappa$ bit-vectors $c_{\pi}^{g}$, so that $c_{\pi}^{g}\left[\operatorname{rank}_{g}\left(c_{\pi}^{\prime}, i\right)\right]=1$ iff $c_{\pi}[i]=g$ (and 0 iff $c_{\pi}[i]=g^{\prime}$ ). We use a representation for $c_{\pi}^{\prime}$ that requires $O(|\pi| \kappa \lg \kappa)$ bits and gives constant select time (Lemma 4). We also add the structure of Lemma 7 to $c_{\pi}^{\prime}$. This adds $O(|\pi| \kappa \lg \lg \kappa)$ bits and allows us to compute $r=\operatorname{rank}_{g}\left(c_{\pi}^{\prime}, i\right)$ in constant time, given that $c_{\pi}^{\prime}[i]=g$. Then we find the latest 1 in $c_{\pi}^{g}[1, r], o=\operatorname{select}_{1}\left(c_{\pi}^{g}, \operatorname{rank}_{1}\left(c_{\pi}^{g}, r\right)\right)$. This corresponds to the last occurrence of $g$ preceding $c_{\pi}[i]=g^{\prime}$. The position is mapped back from $c_{\pi}^{g}[o]$ to $c_{\pi}$ with $j=\operatorname{select}_{g}\left(c_{\pi}^{\prime}, o\right)$.

Lemma 21 Constant-time access to the position $P_{v}[i]$ of any inherited point in the unmarked nodes $v$ can be provided within $O(n \lg \kappa)$ bits of space.

Proof. We have already explained how to obtain the position in constant time. The space is dominated by the sequences $c_{v}$, represented as $c_{\pi}$ and these in turn as $c_{\pi}^{\prime}$ and $c_{\pi}^{g}$, which add up to $O(n \lg \kappa)$ bits. Bit-vectors $B$ and $R$ use just $O(n / \kappa)$ further bits.

Lemmas 19, 20, and 21 prove Theorem 5 . 


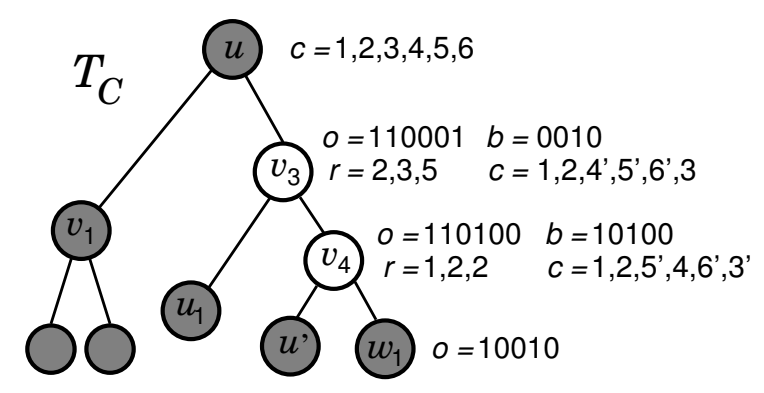

$$
\begin{aligned}
& c= \text { colors } \\
& o= \text { which points are original }(1 \mathrm{~s}) \\
& b= \text { distances to nodes } \mathrm{v}^{\prime} \text { where } \\
& \text { original points appear explicitly } \\
& r= \text { positions where each original } \\
& \text { point appear explicitly in its v' }
\end{aligned}
$$

Figure 5: Illustration of the structures for constant-time access to $P_{v}$ on $T_{C}$, assuming that nodes $v_{3}, v_{4}$, and $u^{\prime}$ are all of the same level. Marked nodes are filled with gray.

Example. In the tree $T_{C}$ in Figure 2, nodes $v_{2}$ and $v_{3}$ are of level 1 and the rest are of level 2, and all turn out to be marked. To show a more interesting example, Figure 5 assumes that the grid has more points towards the bottom, so that the leaves that descended from $v_{3}$ and $v_{4}$ are now internal nodes (and have new labels $u_{1}, u^{\prime}$, and $w_{1}$, whose reason will be clear later), so that nodes $v_{3}, v_{4}$, and $u^{\prime}$ are all of the same level $\ell$, whereas $u_{1}$ and $w_{1}$ are of level $\ell+1$. Then the path is $\pi=\left\langle v_{3}, v_{4}\right\rangle$, node $u$ (which was $v_{2}$ in Figure 2) is the upper limit of $\pi$, and node $u^{\prime}$ acts as its lower limit. For example, $o_{v_{3}}=110001$ because the first, second, and sixth points in the slab of $v_{3}$, read left to right, are original, whereas the others are inherited from $u$. Also, $b_{v_{3}}=0010$, indicating $d_{1}=1, d_{2}=1$, and $d_{3}=2$, because the first and second original points are inherited by $u_{1}$, which is the node at distance 1 that leaves $\pi$. Instead, the third original point of $v_{3}$ is inherited by $w_{1}$, which is the node at distance 2 from $v_{3}$ that leaves $\pi\left(u^{\prime}\right.$ is also at distance 2 ; to avoid ambiguities we assume it is at distance 3 , as explained soon). The positions where those original points are represented in the marked nodes that leave the path are $r_{v_{3}}=2,3,5$, since the first and second are the second and third points in $u_{1}$, and the third original point of $v_{3}$ is the fifth point in $w_{1}$. Finally, $c_{v_{3}}=1,2,4^{\prime}, 5^{\prime}, 6^{\prime}, 3$ because (as shown in $o_{v_{3}}$ ), the third, fourth, and fifth points in $v_{3}$ are inherited, and they correspond to the original points marked 4,5 , and 6 , in the parent $u$. The three new original colors of $v_{3}$ receive arbitrary free colors 1,2 , and 3 . In $v_{4}$, three points (with colors $5^{\prime}, 6^{\prime}$, and $3^{\prime}$ ) are inherited, corresponding to those with colors $5^{\prime}$ and $6^{\prime}$ in $v_{3}$ (which are in turn inherited from 5 and 6 in $u$ ), and to the one with color 3 in $v_{3}$, which is original in that node. The other three colors in $v_{4}$ are original and receive free original colors 1,2 , and 4 . We also show the array $o_{w_{1}}$, since later in the article marked nodes will also store these bit-vectors.

\section{Predecessor queries on $E_{v}$}

Having constant-time access to $E_{v}$ enables searching for the desired limits where the queries are to be run. Recall that our queries involve a range $A[i, j]$ and, for a suitable node $v \in T_{C}$, this translates into finding the largest $l$ and the smallest $r$ such that $E_{v}[l-1]<i \leq j<E_{v}[r+1]$ (see Section 4.2). This is a form of predecessor query on $E_{v}$ that we can perform by a binary search. However the resulting $O(\lg \kappa)$ search time is larger than the promised time complexity. In this section we obtain faster predecessor searches that replace the binary search. Once again, we will focus on providing predecessor searches on $P_{v}$, the positions of the points in the slab of $v$. Predecessors on $E_{v}=P_{v_{-}}: P_{v}: P_{v_{+}}$are then obtained by finding the neighbor nodes $v_{-}$and $v_{+}$, 
as shown in Lemma 15, and then determining with a couple of comparisons whether to run the query on $P_{v-}, P_{v}$, or $P_{v+}$. Concretely, in this section we prove the following theorem:

Theorem 6 Given the structures for constant-time navigation in $T_{C}$ (Lemma 9), for handling shallow cuttings in $T_{C}$ (Lemmas 13 to 16), and for constant-time access to all arrays $P_{v}$ in $T_{C}$ (Theorem 5), predecessor queries on the array $P_{v}$ of any node $v \in T_{C}$ can be carried out in time $O(1+\lg \kappa / \lg \lg n)$ using $O(n \lg \kappa)$ bits of space.

A classical predecessor structure [30] on $P_{v}[1,2 \kappa]$ uses $O(\kappa \lg n)$ bits, as the universe is [1,n], the set of positions in $A$. These spaces would add up to $O(n \lg n)$ bits (note that this structure is needed in all the $O(t)$ nodes of $T_{C}$, not only the marked ones). Instead, since we have independent constant-time access to $P_{v}$, we use succinct SB-trees (Lemma 10).

On a node $v$ of level $\ell$, the universe of positions is of size $O(\kappa|v|)=O\left(\kappa t_{\ell-1}^{2}\right)$, thus the succinct SB-tree would use $O\left(\kappa \lg \lg \left(\kappa t_{\ell-1}^{2}\right)\right)=O\left(\kappa \lg t_{\ell}+\kappa \lg \lg \kappa\right)$ bits. While the second term adds up to $O(n \lg \lg \kappa)$, the first term is still too large: just considering the nodes with $\ell=1$, it adds up to $O(n \lg \lg n)$ bits if we store this structure on every node of $T_{C}$.

To reduce space, we will store this structure only on sampled nodes, and will handle the unsampled ones with other techniques. We will sample all the nodes marked in Section 6 , and in addition we will will further sample every $\left(t_{\ell} / \lg ^{2} t_{\ell}\right)$ th node in the paths $\pi$ of unmarked nodes of level $\ell$. To associate information with sampled nodes of each level, we use the analogous of bitvector $M$ and sequence $L$ of Section 6.2.

Lemma 22 Predecessor queries on the array $P_{v}$ of any sampled node $v$ can be carried out in time $O(1+\lg \kappa / \lg \lg n)$ using $O(n \lg \lg \kappa)$ bits of space.

Proof. According to Lemma 10, the predecessor time with the succinct SB-tree stored at the node is $O\left(1+\lg \kappa / \lg \lg \left(\kappa t_{\ell-1}^{2}\right)\right)$. This can be improved to $O(1+\lg \kappa / \lg \lg n)$ by using the same precomputed table over a universe of size $n$ for all the nodes; this table requires $o(n)$ further bits.

Let us consider space. The number of sampled nodes of level $\ell$ is $O\left(t \lg ^{2} t_{\ell} / t_{\ell}\right)$, which added over all the levels is $\sum_{\ell} t \lg ^{2} t_{\ell} / t_{\ell} \leq t\left(O(1)+\sum_{s>4} s^{2} / 2^{s}\right)=O(t)$ (as in the proof of Lemma 19). Therefore, the term $O(\kappa \lg \lg \kappa)$ in the bit space of succinct SB-trees adds up to $O(n \lg \lg \kappa)$. The other component of the space, $O\left(\kappa \lg t_{\ell}\right)$ bits, adds up to $O\left(n \lg ^{3} t_{\ell} / t_{\ell}\right)$ bits for level $\ell$. Adding up over all the levels $\ell$ we have $O(n) \sum_{\ell} \lg ^{3} t_{\ell} / t_{\ell} \leq O(n)\left(O(1)+\sum_{s>4} s^{3} / 2^{s}\right)=O(n)$ bits. Finally, the analogous of bit-vector $M$ uses $O(t)$ bits and the analogous of $L$ uses $O\left(n_{\ell} \lg \left(|L| / n_{\ell}\right)\right.$ ) bits (recall the proof of Lemma 19). Since now $n_{\ell}=O\left(t \lg ^{2} t_{\ell} / t_{\ell}\right)$, this space is $O(t) \sum_{\ell} \lg ^{3} t_{\ell} / t_{\ell}=O(t)$.

The paths of unsampled nodes of level $\ell$ have length $O\left(t_{\ell} / \lg ^{2} t_{\ell}\right)$. To provide predecessor searches on unsampled nodes, let us consider one such path $\pi$ and let $v$ be a node in $\pi$. The nodes leaving the path are of level $>\ell$, except the node $u^{\prime}$ leaving $\pi$ at the bottom, which is of level $\ell$. Therefore, we can divide the range of split points covered by $\pi$ into three areas:

1. The area covered by the subtrees that leave $\pi$ to the left.

2. The area covered by the subtrees that leave $\pi$ to the right.

3. The area covered by $u^{\prime}$. 
Each of those areas is contiguous, (1) preceding (3) preceding (2). Since there are $O\left(t_{\ell}\right)$ subtrees of type (1) and each has nodes of level at least $\ell+1$, the total area covered by those subtrees is of size $O\left(t_{\ell} \cdot \kappa t_{\ell}^{2}\right)=O\left(\kappa t_{\ell}^{3}\right)$. The case of (2) is analogous. Area (3), instead, can be significantly larger since $u^{\prime}$ can be of level $\ell$. Our solutions will use these areas in different ways depending on whether $\kappa=\Omega(\lg \lg n)$ or $\kappa=O(\lg \lg n)$. We describe each case separately.

\subsection{Handling large $\kappa$ values}

When $\kappa=\Omega(\lg \lg n)$, we can afford to store, for each (unsampled) node $v \in \pi$, a succinct SBtree for the values of $P_{v}$ falling in area (1) and another for the values in area (2), both using $O\left(\kappa \lg \lg \left(\kappa t_{\ell}^{3}\right)\right)=O\left(\kappa \lg \lg \left(\kappa t_{\ell}\right)\right)$ bits. Given a predecessor request on $v$, we first find the node $u^{\prime}$ below $\pi$ as in Section 6.3.1, and determine in constant time whether the query falls in the area (1), (2), or (3) (by obtaining the limits $\left[x_{l}+1, x_{r}\right]$ of $u^{\prime}$, Lemma 16). If the query falls in areas (1) or (2) we use the corresponding succinct SB-tree of $v$, otherwise we use the succinct SB-tree of $u^{\prime}$ (which is sampled and hence stores a regular succinct SB-tree).

While the succinct SB-trees for areas (1) and (2) are built for $v$ and store the positions of the points of $P_{v}$, this is not the case of the regular succinct SB-tree of $u^{\prime}$, since not all the points in $u^{\prime}$ are points in $v$. In this case, given the predecessor $P_{u^{\prime}}[q]$ of a position $p$, we must still find the predecessor of $P_{u^{\prime}}[q]$ in $P_{v}$. The points inherited in $P_{u^{\prime}}$ form a central band in $P_{v}$, starting at position $p_{v}$. Thus we store, for each node $v$, a bit-vector $h_{v}[1,2 \kappa]$ indicating which of the points in its corresponding node $u^{\prime}$ are inherited from $v$, as well as $p_{v}$. Then the final answer is $p_{v}+\operatorname{select}_{1}\left(h_{v}, \operatorname{rank}_{1}\left(h_{v}, q\right)\right)-1$, which is computed in constant time. These arrays add $O(n)$ bits of space.

Example. Figure 6 (left) shows a schematic example of this arrangement. A path $\pi=\left\langle v_{1}, v_{2}, v_{3}, v_{4}\right\rangle$ of level $\ell$ is limited by $u$ and $u^{\prime}$. Nodes $u_{1}$ and $u_{2}$, of level $>\ell$, leave $\pi$ from the left and $w_{1}$ and $w_{2}$, also of level $>\ell$, leave from the right. Node $u^{\prime}$ is of level $\ell$ and is sampled, so it has its own SB-tree. The other nodes leaving $\pi$ cover a smaller area, so we can afford two SB-trees for each $v$, storing the positions of the split points of $P_{v}$ inside the $u_{i}$ nodes and inside the $w_{j}$ nodes. For example, if we build the SB-trees for $v_{2}$, we include in the left succinct SB-tree the positions $P_{v_{2}}$ of the points that are inherited in $\left\langle u_{2}\right\rangle$, and in the the right succinct SB-tree the positions $P_{v_{2}}$ of the points that are inherited in $\left\langle w_{1}, w_{2}\right\rangle$ ( $u_{1}$ cannot have points of $P_{v_{2}}$ because it does not descend from $v_{2}$ ).

Lemma 23 If $\kappa=\Omega(\lg \lg n)$, then predecessor queries in the array $P_{v}$ of any unsampled node $v$ can be carried out in time $O(1+\lg \kappa / \lg \lg n)$ using $O(n \lg \kappa)$ bits of space.

Proof. The time is dominated by the succinct SB-trees, which was explained in Lemma 22. The space of the two additional succinct SB-trees for a node of level $\ell$ is $O\left(\kappa \lg \lg \left(\kappa t_{\ell}\right)\right)$ bits. This adds up to $O(n(\lg \lg \kappa+\lg \lg \lg n))$ bits, the second term being dominated by the (unsampled) nodes of level $\ell=1$. Since $\lg \kappa=\Omega(\lg \lg \lg n)$, the space is bounded by $O(n \lg \kappa)$ bits.

\subsection{Handling small $\kappa$ values}

When $\kappa=O(\lg \lg n)$ we will not store succinct SB-trees for areas (1) and (2) for each unsampled node as before, but will use a different mechanism. Let $\pi$ be a path of unsampled nodes of level 

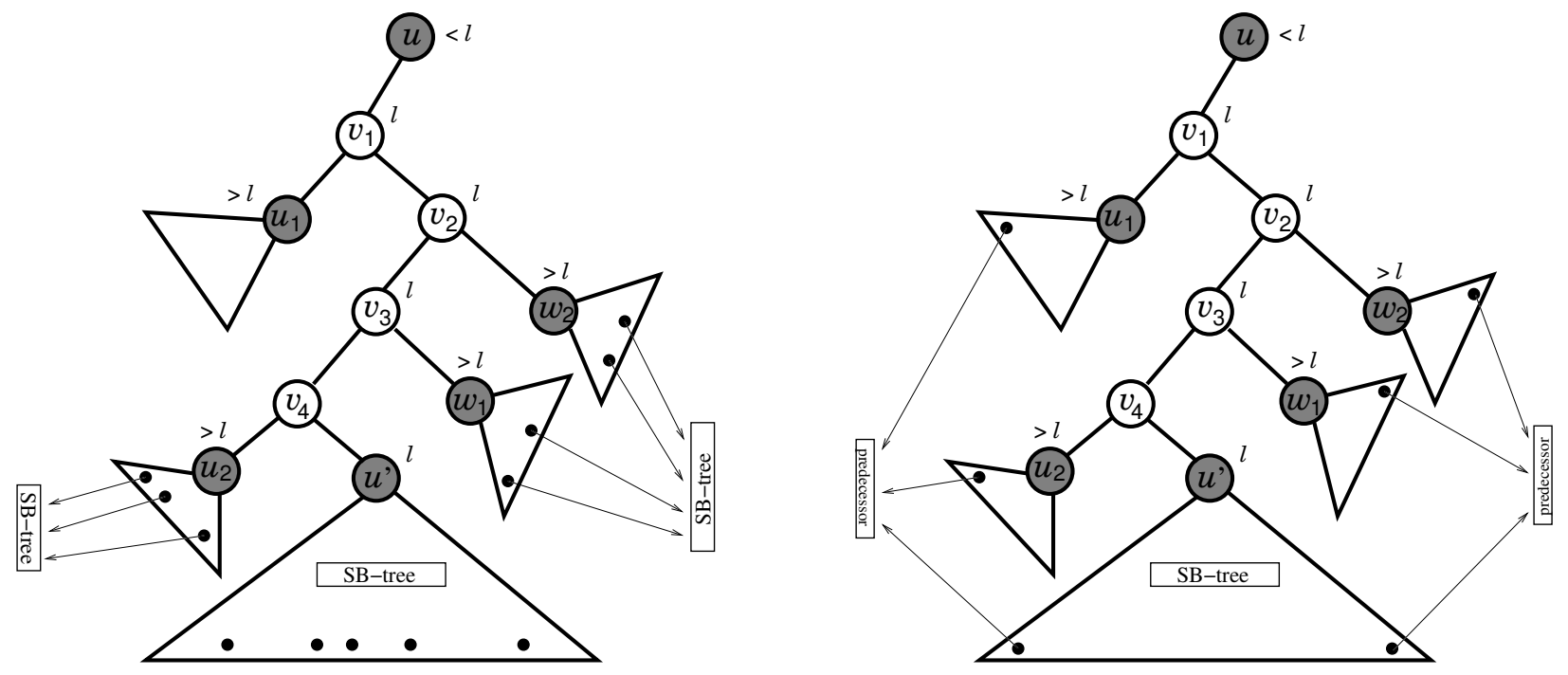

Figure 6: Illustration of the scheme to compute predecessors on paths of unsampled nodes. On the left, the structure for $v_{2}$ when $\kappa=\Omega(\lg \lg n)$. The black dots indicate the points inherited from $v_{2}$. On the right, the structure for the whole path when $\kappa=O(\lg \lg n)$. The black dots indicate the first/last points of the subtree areas.

$\ell$. Let $u_{1}, u_{2}, \ldots$ be the nodes that leave $\pi$ from the left, reading their areas in left-to-right order (i.e., top-down in $\pi$ ) until reaching $u^{\prime}$, and $w_{1}, w_{2}, \ldots$ be the nodes that leave $\pi$ from the right, also reading them in left-to-right order (i.e., bottom-up in $\pi$ ) from $u^{\prime}$. Then the area of $A$ covered by $\pi$ can be partitioned into the $|\pi|+1$ consecutive areas covered by $u_{1}, u_{2}, \ldots, u^{\prime}, w_{1}, w_{2}, \ldots$ All those nodes are sampled and thus store their own succinct SB-trees.

We will use a single predecessor structure, associated with $\pi$ (not with any particular node $v \in \pi$ ), to determine in which of those $|\pi|+1$ areas the query $p$ belongs (if the query is done for a node $v \in \pi$, then the node containing that area will descend from $v$ ).

Let $\ell_{i}$ be the level of node $u_{i}$. Then the area covered by $u_{i}$ is of length $O\left(\kappa t_{\ell_{i}-1}^{2}\right)$. Thus we can encode those lengths with, say, $\gamma$-codes [5], within $2 \sum_{i} \lg \left(\kappa t_{\ell_{i}-1}^{2}\right)=O\left(|\pi| \lg \kappa+\sum_{i} t_{\ell_{i}}\right)$ bits. To facilitate decoding this description, we will insert areas of length zero every time $\pi$ goes left (when encoding the areas $u_{i}$ ) or every time $\pi$ goes right (when encoding the areas $w_{j}$ ). This does not change the asymptotic length of the description.

From a space accounting point of view, this space can be afforded because we can charge $O\left(\lg \kappa+t_{\ell_{i}}\right)$ bits to the storage of $u_{i}$. As $u_{i}$ 's level is larger than $\ell$, it is a marked node (see Section 6). Thus there are $O\left(t / t_{\ell_{i}}^{2}\right)$ such nodes overall, each of which will be charged $O\left(t_{\ell_{i}}\right)$ bits only once, from the path $\pi$ it leaves, for a total of $O\left(t / t_{\ell_{i}}\right)$ bits, and this adds up to $O(t)$ bits overall (see the proof of Lemma 19). As for the term $O(\lg \kappa)$, it adds up to $O(t \lg \kappa)$ bits overall.

On the other hand, note that, since $\ell_{i}>\ell$, it holds that $O\left(|\pi| \lg \kappa+\sum_{i} t_{\ell_{i}}\right)=O(|\pi| \lg \kappa+$ $\left.|\pi| \lg t_{\ell}\right)$. Since $|\pi|=O\left(t_{\ell} / \lg ^{2} t_{\ell}\right), t_{\ell}=O(\lg n)$ even for $\ell=1$, and $\kappa=O(\lg \lg n)$, the length is $O(\lg n / \lg \lg n)=o(\lg n)$ bits, and thus the whole description of the $u_{i}$ area lengths fits in $O(1)$ computer words. ${ }^{11}$ Moreover, there are $2^{O(\lg n / \lg \lg n)}$ possible descriptions of area lengths for $u_{1}, u_{2}, \ldots$, and $O\left(|\pi| \kappa t_{\ell_{i}-1}^{2}\right)=o\left(\lg ^{3} n\right)$ possible queries. Thus we can build a global table of

\footnotetext{
${ }^{11}$ This is why we need the $\lg ^{2} t_{\ell}$ term dividing $t_{\ell}$ in the definition of our sampling.
} 
$2^{O(\lg n / \lg \lg n)} \times o\left(\lg ^{3} n\right) \times \lg n=o(n)$ bits storing the answer to every possible query on every possible path. Thus the queries take constant time. We proceed analogously with the areas of $w_{1}, w_{2}, \ldots$..

Now, a predecessor query for the areas $u_{1}, u_{2}, \ldots, u^{\prime}, w_{1}, w_{2}, \ldots$ can be answered as follows: As in Section 7.1, we first determine whether the answer is in $u^{\prime}$ with a constant number of comparisons, and if so, we obtain the answer with the succinct SB-tree of $u^{\prime}$. Otherwise, the answer is in the areas to the left (called $u_{i}$ ) or to the right (called $w_{j}$ ) nodes of $u^{\prime}$. In either case, we use the precomputed tables to determine in constant time the index $i$ (left) or $j$ (right) of the area where the predecessor lies. If the answer is on the left area, we compute $v^{\prime}=u_{i}$ from the index $i$ as in Section 6.3.1: we find the ancestor $a$ of $u^{\prime}$ at depth $\operatorname{depth}(u)+i$, and then $v^{\prime}$ is the child of $a$ that is not in $\pi$ (i.e., is not an ancestor of $u^{\prime}$ ). If the answer is on the right area, we compute $v^{\prime}=w_{j}$ similarly, but now $a$ is the ancestor of $u^{\prime}$ at depth $\operatorname{depth}\left(u^{\prime}\right)-j$. Note that this works because we have inserted the empty areas in the $\gamma$-encoded descriptions.

Example. Figure 6 (right) illustrates the structure for small $\kappa$ values. Now the predecessor structures associated with $\pi$ (i.e., the $\gamma$-encoded descriptions) store only one extreme split point from each node leaving $\pi$. We must insert two empty areas between $u_{1}$ and $u_{2}$, so the index of $u_{2}$ in the $\gamma$-encoded description is actually 4 , and it is indeed the child not in $\pi$ of the ancestor of $u^{\prime}$ at depth $\operatorname{depth}(u)+4$. Similarly, we insert an empty area before $w_{1}$ and one after $w_{2}$. Then the index of $w_{1}$, for example, is 2 , and it is the child not in $\pi$ of the ancestor of $u^{\prime}$ at depth $\operatorname{depth}\left(u^{\prime}\right)-2$.

Now we use the succinct SB-tree of $v^{\prime}$ (which is sampled) to find the position of the predecessor of $p$ in its $P_{v^{\prime}}$ array, $P_{v^{\prime}}[q]$. The final challenge is to map that position in $v^{\prime}$ to the corresponding position in $v$. Note that $P_{v}$ contains only some of the positions of $P_{v^{\prime}}$ in the area covered by $v^{\prime}$ (where $p$ lies), so we seek the predecessor of $P_{v^{\prime}}[q]$ in $P_{v}$. To compute this efficiently, we will reuse the point inheritance information encoded in the bit-arrays $o_{v}$ of Section 6 . With the sequence of $|\pi|$ consecutive arrays $o_{v}$, and knowing whether each node in $\pi$ is a left or a right child, we have sufficient information to track any position $P_{v^{\prime}}[q]$ upwards and determine its predecessor in $P_{v}$.

Let $q^{\prime}=\operatorname{rank}_{0}\left(o_{v^{\prime}}, q\right)$ be the number of inherited points having positions in $P_{v^{\prime}}[1, q]$, and $v^{\prime \prime}$ be the parent of $v^{\prime}$. If $v^{\prime}$ is the left child of $v^{\prime \prime}$, then the first half of the points of $v^{\prime \prime}$ are inherited by $v^{\prime}$, and therefore the position $P_{v^{\prime}}[q]$, or its predecessor, in $v^{\prime \prime}$ is $P_{v^{\prime \prime}}\left[q^{\prime}\right]$ (note that $q^{\prime}$ can be zero). If, instead, $v^{\prime}$ is the right child of $v^{\prime \prime}$, then the position is $P_{v^{\prime \prime}}\left[\kappa+q^{\prime}\right]$ since $v^{\prime}$ inherits the second half of the points. Now we repeat the process from $v^{\prime \prime}$ until reaching $v$, where we obtain the final predecessor position in $P_{v}$.

Example. Consider Figure 5 and let $v_{3}$ have no SB-tree of its own. Assume that a predecessor search in $v_{3}$ is found to fall inside the node $w_{1}$. Since $w_{1}$ is marked (and thus sampled), it has its own SB-tree, which is searched to find the predecessor, $P_{w_{1}}[2]$ (this is the 17 th left-to-right point in Figure 2). Since $o_{w_{1}}[2]=0$, the point is inherited. It is the first inherited point because rank $_{0}\left(o_{w_{1}}, 2\right)=1$. Since $w_{1}$ is a right child and $\kappa=3$, the point is the fourth $(3+1)$ in $v_{4}$. The point is original in $v_{4}$, since $o_{v_{4}}[4]=1$. The number of inherited points in $v_{4}$ preceding this original point is $\operatorname{rank}_{0}\left(o_{v_{4}}, 4\right)=1$, so the first inherited point is the predecessor in $v_{3}$, the parent of $v_{4}$. Since $v_{4}$ is a right child, the predecessor is the fourth left-to-right point $(3+1)$ in the slab of $v_{3}$.

To use the bit-vectors $o_{v}$ in this way, we cannot use the same array $o[1, O(n)]$ where they were stored in preorder in Section 6.3.1. Rather, we must store another copy of bit-vectors $o_{v}$ in the 
path-wise form used to store the sequences $c_{v}$ in Section 6.3.2, so that all the bit-vectors $o_{v}$ for unsampled nodes $v \in \pi$ are stored contiguously in a sequence $o_{\pi}$. In addition, we need the bitvectors $o_{v^{\prime}}$ for sampled nodes $v^{\prime}$. Sampled nodes can be handled as belonging to an empty path where the sampled node acts as $u^{\prime}$, and we also store $o_{u^{\prime}}$ in $o_{\pi}$. The space for this new copy of the $o_{v}$ bit-vectors is $O(n)$ bits. We similarly store the information on left/right directions along each path $\pi$, contiguously and adding up to $O(t)$ bits.

Now the bit-vectors $o_{v}$ and the path directions along $\pi$ are stored contiguously and add up to length $2|\pi| \kappa$ and $|\pi|$, respectively. Thus, once again, we can prepare a global table that takes every possible concatenation of bit-vectors $o_{\pi}$, a bit-vector $o_{v^{\prime}}$, the $|\pi|$ left/right directions along the path $\pi$, the depths of $v$ and $v^{\prime}$ in $\pi$, and the value $q$, and it returns the corresponding predecessor

in $P_{v}$ in constant time. The table uses $2^{(|\pi|+1)(2 \kappa+1)}|\pi|^{2} \kappa \cdot \lg n$ bits. Since $\kappa=O(\lg \lg n)$, this is $2^{O(\lg n / \lg \lg n)} o\left(\lg ^{3} n\right)=o(n)$ bits.

Lemma 24 If $\kappa=O(\lg \lg n)$, then predecessor queries in the $P_{v}$ array of any unsampled node $v$ can be carried out in time $O(1+\lg \kappa / \lg \lg n)$ using $O((n / \kappa) \lg \kappa)+o(n)$ bits of space.

Proof. The time is again dominated by the succinct SB-tree of $u^{\prime}$, which was explained in Lemma 22. The space is that of the $\gamma$-encoded descriptions and global tables.

Lemmas 22, 24, and 23 complete the proof of Theorem 6 .

\section{Wrapping up}

From the previous elements, we can now assemble a structure that, given a value $\kappa$, uses $O(n \lg \kappa)$ bits and answers a query $\operatorname{sel}(i, j, k)$ for any $1 \leq k \leq \kappa$ in time $O(1+\lg \kappa / \lg \lg n)$ :

1. As described in Section 5 (Lemma 12), we find the maximal interval $[m, M]$ such that $i \leq$ $x_{m} \leq x_{M} \leq j$.

2. If the interval contains zero or one split point, then $A[i, j]$ can be directly solved with the range selection structure [8] associated with the special extent $\left[x_{m-1}+1, x_{m+1}\right]$ of the split point $x_{m}$, which covers at most $4 \kappa$ consecutive entries of $A$.

3. Otherwise, we find the highest node $v \in T_{C}$ containing $\left[x_{m}+1, x_{M}\right]$, as well as the other two neighbor nodes that span the extent of $v$, namely, $v_{-}$to the left and $v_{+}$to the right, all in constant time, as described in Section 5 (Lemmas 13, 14, and 15).

4. Using the structures of Section 7 (Theorem 6), we find the predecessor $l-1$ of $i-1$, and the predecessor $r$ of $j$, within the positions of $E_{v}=P_{v_{-}}: P_{v}: P_{v_{+}}$, in time $O(1+\lg \kappa / \lg \lg n)$. These structures need access to entries in $P_{v_{-}}, P_{v}$, and $P_{v_{+}}$, which is provided in constant time in Section 6 (Theorem 5).

5. We use the range selection structure $[8,9]$ associated with the extent of node $v$ (which has at most $6 \kappa$ entries) to run the query $o=\operatorname{sel}(l, r, k)$. The time is $O(1+\lg \kappa / \lg \lg n)$.

6. We use the structures of Section 6 (Theorem 5) to compute the final answer $E_{v}[o]$ in constant time, which is again provided via direct access to arrays $P_{v_{-}}, P_{v}$, or $P_{v_{+}}$. 
In order to reduce the time from $O(1+\lg \kappa / \lg \lg n)$ to $O(1+\lg k / \lg \lg n)$, we build our data structures for values $\kappa_{s}=2^{2^{s}}$, for $s=0,1, \ldots, \tau$, where $\tau$ is such that $2^{2^{\tau-1}}<\kappa \leq 2^{2^{\tau}}$. The space for those structures is $O(n) \sum_{s=0}^{\tau} \lg \kappa_{s}=O(n) \sum_{s=0}^{\tau} 2^{s}<O(n) 2^{\tau+1}<O(n) 4 \lg \kappa=O(n \lg \kappa)$. A query $\operatorname{sel}(i, j, k)$ is run on the structure for $\kappa_{s}$ such that $\kappa_{s-1}<k \leq \kappa_{s}$, that is, $2^{s-1}<\lg k \leq 2^{s},{ }^{12}$ and thus its query time is $O\left(1+\lg \kappa_{s} / \lg \lg n\right)=O\left(1+2^{s} / \lg \lg n\right)=O(1+\lg k / \lg \lg n)$. This proves Theorem 2.

\subsection{Answering the query top $(i, j, k)$}

To answer a query top $(i, j, k)$ we can proceed as for query sel $(i, j, k)$, until the point where we find the $k$ th largest element in $A_{v}[l, r]$, let it be $A_{v}[o]$. Now we find all the elements $A_{v}[m]$ in $A_{v}[l, r]$ where $A_{v}[m] \geq A_{v}[o]$. With an rmq structure over $A_{v}$ we can do this using Muthukrishnan's algorithm [27]: find the maximum in $A_{v}[l, r]$, let it be $A_{v}\left[m_{1}\right]$, then continue recursively with $A_{v}\left[l, m_{1}-1\right]$ and $A_{v}\left[m_{1}+1, r\right]$ stopping the recursion when the maximum, found at $A_{v}[m]$, satisfies $A_{v}[m]<A_{v}[o]$. Recall that $A_{v}$ is a permutation on $O(\kappa)$ symbols and thus we can afford storing it directly (actually, it is generally part of the selection structures we use [8]). Finally, when we have the positions $m_{1}, \ldots, m_{k}$ of the top- $k$ elements, we return $E_{v}\left[m_{1}\right], \ldots, E_{v}\left[m_{k}\right]$. The overall time is $O(\lg k / \lg \lg n+k)=O(k)$.

Note that this process delivers the top- $k$ elements in arbitrary order. On the other hand, the set is obtained in online form: after $O(1+\lg k / \lg \lg n)$ time, each new result is delivered in $O(1)$ time. To obtain the result in sorted order and in online form, we build the structure of Brodal et al. [6] on the sets $A_{v}$, which amounts to $O(n \lg \kappa)$ further bits. With this structure, we retrieve the $k$ highest values of $A_{v}[l, r]$ in time $O(k)$ and in online form, analogously as what is done with the structure of Brodal and Jørgensen [8] for the query sel(·). This proves Theorem 3.

\section{One-sided queries}

We finish by showing that, at least in some restricted cases that might be of interest, the time lower bound for sel(.) queries can be circumvented. We will design an encoding that is built for a fixed $\kappa$ value and answers queries sel $(1, j, \kappa)$ and top $(1, j, \kappa)$. We start with the following result for sel $(\cdot)$ queries, and then use the same encoding to solve top $(\cdot)$ queries.

Theorem 7 Given an array $A[1, n]$ and a value $\kappa$, there are encodings of $A$ and $\kappa$ that (1) use $n \lg \kappa+o(n \lg \kappa)+n$ bits and support $\operatorname{sel}(1, j, \kappa)$ queries in any $\omega(1)$ time, or $(2)$ use $(1+\epsilon) n \lg \kappa$ bits and support $\operatorname{sel}(1, j, \kappa)$ queries in $O(1 / \epsilon)$ time, for any constant $0<\epsilon<1$.

To build this encoding, we scan the array from left to right, and keep track of the top- $\kappa$ values in the prefix seen so far. At any position $j>\kappa$, if $A[j]$ is inserted into the top- $\kappa$ list, then we have to remove the $\kappa$ th largest value in the prefix $A[1, j-1]$. The idea to solve these queries is to record the position of that leaving $\kappa$ th largest value, so that to solve $\operatorname{sel}(1, j, \kappa)$ we find the next $j^{\prime}>j$ where the top- $\kappa$ list changes, and then find the value leaving the list when $A\left[j^{\prime}\right]$ enters it. This one was the $\kappa$ th largest value in $A[1, j]$.

We wish, however, to store this information using only $O(n \lg \kappa)$ bits. The key idea is to store colors in $[1, \kappa]$ associated with the positions $A\left[j^{\prime}\right]$ where the top- $\kappa$ list changes. Each element that

\footnotetext{
${ }^{12}$ The search for the right $s$ can be done in constant time by checking the cases $s=\tau$ and $s=\tau-1$, and then consulting a small precomputed table of $2^{2^{\tau-2}}=O(\sqrt{\kappa})$ entries.
} 


$\begin{array}{lllllllllllllllllll}\text { A } & 12 & 18 & 17 & 20 & 14 & 19 & 22 & 11 & 25 & 21 & 28 & 16 & 23 & 13 & 15 & 24 & 29 & 27 \\ \text { X } & 1 & 2 & 3 & 1 & - & 3 & 2 & - & 3 & 1 & 1 & - & 2 & - & - & 2 & 2 & 3 \\ \text { P } & 1 & 1 & 1 & 1 & 0 & 1 & 1 & 0 & 1 & 1 & 1 & 0 & 1 & 0 & 0 & 1 & 1 & 1\end{array}$

Figure 7: Encoding of an array $A$ as $P$ and $X$, to support sel( $\cdot)$ and top $(\cdot)$ queries, for $\kappa=3$.

enters the list takes the color of the element leaving it. Then, for every prefix $A[1, j]$, the rightmost positions of the $\kappa$ different colors in $[1, j]$ form the top- $\kappa$ list for $A[1, j]$. In particular, if $A\left[j^{\prime}\right]$ is of color $c$, then the rightmost occurrence of $c$ in $A\left[1, j^{\prime}-1\right]$ is the position of the $\kappa$ th element in $A\left[1, j^{\prime}-1\right]$, that is, $\operatorname{sel}\left(1, j^{\prime}-1, \kappa\right)$ (and also sel $(1, j, \kappa)$, since no changes occur in $A\left[j+1, j^{\prime}-1\right]$ ).

We store a bit-vector $P[1, n]$, where $P[j]=1$ iff a new element is inserted into the top- $\kappa$ list at position $j$ (or equivalently, the $\kappa$ th largest value of $A[1, j-1]$ is deleted at position $j$ ). The first $\kappa$ bits of $P$ are 1 . We encode $P$ in $n+o(n)$ bits supporting constant-time rank and select (Lemma 1).

Let $n^{\prime}$ be the number of $1 \mathrm{~s}$ in $P$. Our string of colors, $X\left[1, n^{\prime}\right]$, holds $X[j]=j$ for $1 \leq j \leq \kappa$, and $X[j]=X\left[\operatorname{rank}_{1}\left(P, \operatorname{sel}\left(1, \operatorname{select}_{1}(P, j)-1\right), \kappa\right)\right]$ for $\kappa<j \leq n^{\prime}$. Basically, if $A[j]$ becomes part of the top- $\kappa$ list in $A[1, j]$, and this displaces the previous top- $\kappa$ element $A[i]$ of $A[1, j-1]$, then we assign $X[j]=X[i]$. The rest of the formula accounts for the fact that $X$ is defined only on the cells of $A$ where the top- $\kappa$ list changes, that is, where $P[j]=1$.

Example. Figure 7 shows an example for an array $A[1,18]$ and $\kappa=3$. The top- $\kappa$ list changes $n^{\prime}=13$ times, so we store $X[1,13]$. The dashes in $X$ are for illustration purposes and are not actually stored; its actual values are associated with the 1 s in $P$.

We encode $X$ in $(1+o(1)) n^{\prime} \lg \kappa$ bits, so that select on $X$ is supported in $O(1)$ time and access to any $X[j]$ takes any $\omega(1)$ time (Lemma 4 ). On top of this we add the structure of Lemma 7 , which uses $O\left(n^{\prime} \lg \lg \kappa\right)=o\left(n^{\prime} \lg \kappa\right) \operatorname{bits}^{13}$ and supports in constant time the restricted queries $\operatorname{rank}_{X[j]}(X, j)$.

Therefore, we compute $i=\operatorname{rank}_{1}(P, j)+1$, and $c=X[i]$ is the color associated with $A\left[j^{\prime}\right]$. Then it holds that $\operatorname{sel}(1, j, \kappa)=\operatorname{select}_{1}\left(P, \operatorname{select}_{c}\left(X, \operatorname{rank}_{c}(X, i)-1\right)\right)$. Thus, this operation can be supported in any $\omega(1)$ time, dominated by the time to access $X[i]$. By using a slightly larger representation for $X$ (Lemma 6 ), $(1+\epsilon) n^{\prime} \lg \kappa$ bits, we obtain time $O(1 / \epsilon)$ for any constant $\epsilon>0$. Theorem 7 follows.

\subsection{Solving top- $\kappa$ queries}

We now use the same encoding to support top $(1, j, \kappa)$ queries.

Theorem 8 Given an array $A[1, n]$ and a value $\kappa$, there is an encoding of $A$ and $\kappa$ that uses $n \lg \kappa+o(n \lg \kappa)+n$ bits and supports top $(1, j, \kappa)$ queries in $O(\kappa)$ time, giving the results in unsorted order. The result can be sorted by value in $O(\kappa \lg \lg \kappa)$ time. The encoding is the same as in Theorem 7 .

\footnotetext{
${ }^{13}$ This is $o\left(n^{\prime} \lg \kappa\right)$ only if $\kappa$ is not constant, but if $\kappa=O(1)$ we can directly use the general rank operation of Lemma 4 , which in this case takes constant time.
} 
For supporting top $(\cdot)$ queries we need to find, given a position $X[i]$, the rightmost occurrence preceding $i$ of every color in $[1, \kappa]$. This can be done in $O(\kappa)$ time using the representation of Lemma 4 for $X$ : The string is cut into chunks of size $\kappa$. Each chunk stores an inverted list of its contents, that is, for each color it stores an increasing list of the positions where it appears in the chunk. Constant-time access is given to any position of any list. Further, one bit-vector $B_{c}$ per color $c$ is stored, $B_{c}=01^{n_{1}^{c}} 01^{n_{2}^{c}} \ldots 01_{\kappa}^{n_{\kappa}^{c}}$, where $c$ appears $n_{j}^{c}$ times in the $j$ th chunk. Bit-vectors $B_{c}$ are provided with constant-time rank and select (Lemma 1) and add up to $O\left(n^{\prime}\right)$ bits.

In the chunk $l=\lceil i / \kappa\rceil$ where position $i$ belongs, we traverse all the lists of all the colors, so as to record the last occurrence of each color preceding position $i$. This takes time $O(\kappa)$ because there are $\kappa$ positions in the chunk, thus the total length of the lists is also $\kappa$. Some colors may not occur in the chunk before position $i$, however. For each such color $c$, we find its last position in the last chunk before the current one: the starting point of the chunk $l$ in $B_{c}$ is $s=\operatorname{select}_{0}\left(B_{c}, l\right)$, the number of 1 s up to $s$ is $o=s-l$, and the chunk where the oth 1 appears is $\operatorname{select}_{1}\left(B_{c}, o\right)-o$. Once we find the chunk for $c$ in constant time, we return the last position of the list of $c$ in the chunk, which as said can be accessed in constant time as well.

By the definition of $X$, it is clear that the rightmost occurrences, up to position $i=\operatorname{rank}_{1}(P, j)$, of the distinct colors, form precisely the answer to top $(1, j, \kappa)$. Thus we find all those positions $p$ in time $O(\kappa)$ and remap them to the original array with $\operatorname{select}_{1}(P, p)$.

Note that the top- $\kappa$ positions do not come sorted by value. By the same properties of $X$, if the first occurrence of $c$ after $X[i]$ precedes the first occurrence of $c^{\prime}$ after $X[i]$, then the value associated with $c$ in our answer is smaller than that associated with $c^{\prime}$, as it is replaced earlier. Thus we find the first occurrence, after $i$, of each color $c$ in $[1, \kappa]$. The number $r_{c}$ of times $c$ appears up to position $i$ is select ${ }_{0}\left(B_{c}, l\right)-l$ plus the number of its occurrences up to $i$ inside chunk $l$, which we have already counted. Then the position of its next occurrence in $X$ is $p_{c}=\operatorname{select}_{c}\left(X, r_{c}+1\right)$, which is computed in constant time in our representation of $X$ (Lemma 4). Once we have the positions $p_{c}$, which are integers in $\left[1, n^{\prime}\right]$, we can sort them in time $O(\kappa \lg \lg \kappa)$ [1].

Actually, the space $(1+o(1)) n^{\prime} \lg \kappa$ given in Lemma 4 is obtained using the chunks structure only when $\kappa=\omega(1)$. When $\kappa=O(1)$ ones uses instead Lemma 5 , where operations access, rank $\mathrm{k}_{c}$ and select ${ }_{c}$ on $X$ take constant time. In this case we simply obtain the last position of $c$ before $X[i]$ with select $c\left(X, \operatorname{rank}_{c}(X, i-1)\right)$, and the position following $X[i]$ with $\operatorname{select}_{c}\left(X, \operatorname{rank}_{c}(X, i)+1\right)$, all in constant time per color. Theorem 8 follows.

\section{Conclusions}

We have studied for the first time the problem of encoding data structures for array range queries $\operatorname{sel}(\cdot)$ and $\operatorname{top}(\cdot)$, which return the $k$ th largest element or all the top- $k$ elements, respectively, of any interval $A[i, j]$. An encoding data structure cannot access the array $A$. We have shown that at least $n \lg k-O(n+k \lg k)$ bits are necessary for any such encoding. Further, we have given $O(n \lg \kappa)$-bit encodings that answer both queries, for any $1 \leq k \leq \kappa$, in optimal times $O(1+\lg k / \lg \lg n)$ and $O(k)$, respectively.

A recent followup work [18] refines our lower bound to $(n \lg k+(k+1) n \lg (1+1 / k))(1-o(1))$ bits for $k=o(n)$, and proves it is tight up to lower-order terms by building an encoding of $n \lg \kappa+O(n)$ bits for queries with a fixed $\kappa$ value. The encoding does not, however, support efficient queries; it requires $\Omega(n)$ time. In the most recent version [17], they give a slightly larger encoding using $1.5 n \lg \kappa-\Theta(n)$ bits, which solves queries top $(i, j, \kappa)$ and $\operatorname{sel}(i, j, \kappa)$ in time $O\left(\kappa^{6} \lg ^{2} n \omega(1)\right)$. While 
still far from optimal, the time is polynomial in $\kappa \lg n$ and raises the question of what the space/time tradeoffs are when we consider the constant accompanying the $O(n \lg \kappa)$ space complexity of the encodings. Our encoding obtains optimal times, but the constant is large: $44 n \lg \kappa+O(n \lg \lg \kappa)$ bits plus 32 times the space used by the extra structures $[6,8]$.

Acknowledgements. We thank Yakov Nekrich, who pointed us the results of Brodal et al. [6], and the anonymous referees for their suggestions.

\section{References}

[1] A. Andersson, T. Hagerup, S. Nilsson, and R. Raman. Sorting in linear time? Journal of Computer and System Sciences, 57(1):74-93, 1998.

[2] D. Belazzougui, P. Boldi, R. Pagh, and S. Vigna. Monotone minimal perfect hashing: searching a sorted table with $O(1)$ accesses. In Proc. 20th Annual ACM-SIAM Symposium on Discrete Algorithms (SODA), pages 785-794, 2009.

[3] D. Belazzougui and G. Navarro. Alphabet-independent compressed text indexing. ACM Transactions on Algorithms (TALG), 10(4):article 23, 2014.

[4] D. Belazzougui and G. Navarro. Optimal lower and upper bounds for representing sequences. ACM Transactions on Algorithms, 11(4):article 31, 2015.

[5] T. Bell, J. Cleary, and I. Witten. Text compression. Prentice Hall, 1990.

[6] G. S. Brodal, R. Fagerberg, M. Greve, and A. Lopez-Ortiz. Online sorted range reporting. In Proc. 20th International Symposium on Algorithms and Computation (ISAAC), LNCS 5878, pages 173-182, 2009.

[7] G. S. Brodal, B. Gfeller, A. G. Jørgensen, and P. Sanders. Towards optimal range medians. Theoretical Computer Science, 412(24):2588-2601, 2011.

[8] G. S. Brodal and A. G. Jørgensen. Data structures for range median queries. In Proc. 20th International Symposium on Algorithms and Computation (ISAAC), LNCS 5878, pages 822831, 2009.

[9] T. Chan and B. T. Wilkinson. Adaptive and approximate orthogonal range counting. In Proc. 24th Annual ACM-SIAM Symposium on Discrete Algorithms (SODA), pages 241-251, 2013.

[10] D. Clark. Compact Pat Trees. PhD thesis, University of Waterloo, Canada, 1996.

[11] P. Davoodi, G. Navarro, R. Raman, and S. Srinivasa Rao. Encoding range minima and range top-2 queries. Philosophical Transactions of the Royal Society A, 372(20130131), 2014.

[12] J. Fischer. Optimal succinctness for range minimum queries. In Proc. 9th Latin American Symposium on Theoretical Informatics (LATIN), pages 158-169, 2010.

[13] J. Fischer. Combined data structure for previous- and next-smaller-values. Theoretical Computer Science, 412(22):2451-2456, 2011. 
[14] J. Fischer and V. Heun. Space-efficient preprocessing schemes for range minimum queries on static arrays. SIAM Journal of Computing, 40(2):465-492, 2011.

[15] T. Gagie, G. Navarro, and S. J. Puglisi. New algorithms on wavelet trees and applications to information retrieval. Theoretical Computer Science, 426-427:25-41, 2012.

[16] T. Gagie, S. J. Puglisi, and A. Turpin. Range quantile queries: another virtue of wavelet trees. In Proc. 16th International Symposium on String Processing and Information Retrieval (SPIRE), LNCS 5721, pages 1-6, 2009.

[17] P. Gawrychowski and P. K. Nicholson. Optimal encodings for range min-max and top-k. CoRR, 1411.6581v2, 2015. http://arxiv.org/abs/1411.6581v2.

[18] P. Gawrychowski and P. K. Nicholson. Optimal encodings for range top-k, selection, and minmax. In Proc. 42nd International Colloquium on Automata, Languages, and Programming (ICALP), Part I, LNCS 9134, pages 593-604, 2015.

[19] A. Golynski, I. Munro, and S. Rao. Rank/select operations on large alphabets: a tool for text indexing. In Proc. 17th Annual ACM-SIAM Symposium on Discrete Algorithms (SODA), pages 368-373, 2006.

[20] R. Grossi, J. Iacono, G. Navarro, R. Raman, and S. Srinivasa Rao. Encodings for range selection and top- $k$ queries. In Proc. 21st Annual European Symposium on Algorithms (ESA), LNCS 8125, pages 553-564, 2013.

[21] R. Grossi, A. Orlandi, R. Raman, and S. S. Rao. More haste, less waste: Lowering the redundancy in fully indexable dictionaries. In Proc. 26th International Symposium on Theoretical Aspects of Computer Science (STACS), LIPIcs 3, pages 517-528, 2009.

[22] P. Hsu and G. Ottaviano. Space-efficient data structures for top-k completion. In World Wide Web Conference (WWW 2013), pages 583-594, 2013.

[23] A. G. Jørgensen and K. G. Larsen. Range selection and median: Tight cell probe lower bounds and adaptive data structures. In Proc. 22nd Annual ACM-SIAM Symposium on Discrete Algorithms (SODA), pages 805-813, 2011.

[24] G. Li, S. Ji, C. Li, and J. Feng. Efficient type-ahead search on relational data: a tastier approach. In U. Çetintemel, S. B. Zdonik, D. Kossmann, and N. Tatbul, editors, SIGMOD Conference, pages 695-706. ACM, 2009.

[25] Jirí Matousek. Cutting hyperplane arrangements. Discrete and Computational Geometry, 6:385-406, 1991.

[26] J. I. Munro and V. Raman. Succinct representation of balanced parentheses and static trees. SIAM Journal on Computing, 31(3):762-776, 2001.

[27] S. Muthukrishnan. Efficient algorithms for document retrieval problems. In Proc. 13th Annual ACM-SIAM Symposium on Discrete Algorithms (SODA), pages 657-666, 2002. 
[28] G. Navarro, R. Raman, and S. Srinivasa Rao. Asymptotically optimal encodings for range selection. In Proc. 34th Annual Conference on Foundations of Software Technology and Theoretical Computer Science (FSTTCS), pages 291-302, 2014.

[29] G. Navarro and K. Sadakane. Fully-functional static and dynamic succinct trees. ACM Transactions on Algorithms, 10(3):article 16, 2014.

[30] M. Pătraşcu and M. Thorup. Time-space trade-offs for predecessor search. In Proc. 38th Annual ACM Symposium on Theory of Computing (STOC), pages 232-240, 2006.

[31] R. Raman, V. Raman, and S. Srinivasa Rao. Succinct indexable dictionaries with applications to encoding $k$-ary trees, prefix sums and multisets. ACM Transactions on Algorithms, 2(4):article 43, 2007.

[32] K. Sadakane. Succinct representations of lcp information and improvements in the compressed suffix arrays. In Proc. 13th Annual ACM-SIAM Symposium on Discrete Algorithms (SODA), pages 225-232, 2002. 